ADIFOR Working Note \#7

Extending Compile-Time Reverse Mode and Exploiting Partial Separability in ADIFOR

by

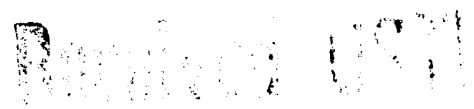

056219992

Christian H. Bischof and Moe El-Khadiri

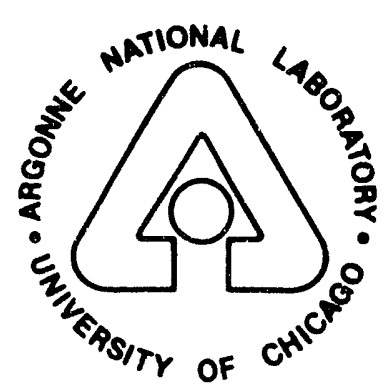
DIVISION 
Argonne National Laboratory, with facilities in the states of Illinois and Idaho, is owned by the United States government, and operated by The University of Chicago under the provisions of a contract with the Department of Energy.

DISCLAIMER

This report was prepared as an account of work sponsored by an agency of the United States Government. Neither the United States Government nor any agency thereot, nor any of their employees, makes any warranty, express or implied, or assumes any legal liability or responsibility for the accuracy, completeness, or usefulness of any information, apparatus, product, or process disclosed, or represents that its use would not infringe privately owned rights. Reference herein to any specific commercial product, process, or service by trade name, trademark, manufacturer, or otherwise, does not necessarily constitute or imply its endorsement, recommendation, or favoring by the United States Government or any agency thereof. The views and opinions of authors expressed herein do not necessarily state or reflect those of the United States Government or any agency thereof.

Reproduced from the best available copy.

Available to DOE and DOE contractors from the Office of Scientific and Technical Information

P.O. Box 62

Oak Ridge, TN 37831

Prices available from (615) 576-8401

Available to the public from the National Technical Information Service

U.S. Department of Commerce 5285 Port Royal Road Springfield. VA 22161 
ARGONNE NATIONAL LABORATORY

9700 South Cass Avenue

Argonne, Illinois 60439
ANL/MCS-TM-163

DE93 004668

\section{ANL/MCS-TM-163 \\ ADIFOR Working Note \#7: \\ Extending Compile-Time Reverse Mode and Exploiting Partial Separability in ADIFOR}

by

Christian H. Bischof and Moe El-Khadiri

Mathematics and Computer Science Division

Technical Memorandum No. 163

October 1992

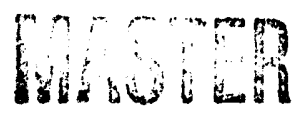

'This work was supported by the Applied Mathematical Sciences subprogram of the Office of Energy Rrsparch, U.S. Department of Energy, under Contract W-31-109-Eng-38. 


\section{Contents}

$\begin{array}{ll}\text { Abstract } & 1\end{array}$

1 Introduction 1

2 Current ADIFOR Strategy 3

3 Extending the Scope of the Compile-Time Reverse Mode 7

3.1 ('ase 1: Reverse Mode for Basic Blocks inside the Loop . . . . . . . . . . . . . . 7

3.2 (ase 2 : Reverse Mode for the Whole Loop . . . . . . . . . . . . . . . . . 7

3.3 ('ase 3 : The Full Reverse Mode . . . . . . . . . . . . . . . . 9 9

4 Exploring Partial Separability 11

5 Experimental Results 16

$\begin{array}{ll}\text { Acknowledgments } & 16\end{array}$

$\begin{array}{ll}\text { References } & 16\end{array}$

APPENDICES: Code Listings for the Torsion Problem 19

A Minpack-2 Code for the Torsion Problem 19

B Current ADIFOR Code for Torsion Problem 21

C Reverse Mode for Basic Blocks

D Reverse Mode for Loop Bodies $\quad 27$

E Reverse Mode for the Whole Program 30

F Derivative Code for Component Functions $\quad 32$

G Enhanced Derivative Code for Component Functions 37 


\title{
ADIFOR Working Note \#7: \\ Extending Compile-Time Reverse Mode and Exploiting Partial Separability in ADIFOR
}

by

\author{
Christian H. Bischof and Moe EL-Khadiri
}

\begin{abstract}
The numerical methods employed in the solution of many scientific computing problems require the computation of the gradient of a function $f: \mathbf{R}^{n} \rightarrow \mathbf{R}$. ADIFOR is a source translator that, given a collection of subroutines to compute $f$, generates Fortran 77 code for computing the derivative of this function. Using the so-called iorsion problem from the MINPACK-2 test collection as an example, this paper explores two issues in automatic differentiation: the efficient computation of derivatives for partial separable functions and the use of the compile-time reverse mode for the generation of derivatives. We show that orders of magnitudes of improvement are possilje: whicn exploiting partial separability and maximizing use of the reverse mode.
\end{abstract}

\section{Introduction}

Differentiation is one of the most fundamental mathematical concepts. In system analysis and control, the investigation into the effect of a disturbance or a change in design parameters on the performance of the overall systern is essential. Mathematically, the change can be modeled by the derivative of the system output with respect to a design parameter. Another application is the numerical solution of initial value problems in stiff ordinary differential equations (see, for example [7, 18]). Methods such as implicit Runge-Kutta and backward differentiation formula (BDF) methods require a Jacobian which is either supplied by the user or approximated by finite differences. In the context of optimization, one needs the derivatives of the objective function. For example, given a function

$$
f: \mathbf{R}^{n} \rightarrow \mathbf{R}
$$

one can find a minimizer $x_{*}$ of $f$ using variable metric methods that involve the iteration

$$
\begin{aligned}
& \text { for } i=1,2, \ldots \text { do } \\
& \quad \text { Solve } B_{i} s_{i}=-\nabla f\left(x_{i}\right) \\
& \quad x_{i+1}=x_{i}+\alpha_{i} s_{i} \\
& \text { end for }
\end{aligned}
$$

where $B_{i}$ is a symmetric positive definite matrix approximating the Hessian of $f$ at $x_{i}$.

These methods are examples of typical methods applied in numerical computations, where the computation of the derivative is a crucial step in the numerical solution process $([6,9,12,8,21])$. One particular optimization problem is the elastic plastic torsion problem, which arises from the determination of the stress field on an infinitely long cylindrical bar. The infinite-dimensional version of this problem is of the form

$$
\min \{g(v): v \in K\}
$$

where $q: K \rightarrow R$ is the quadratic

$$
q(v)=\frac{1}{2} \int_{D}\|\nabla v(x)\|^{2} d x-c \int_{D} v(x) d x
$$

for some constant $c$, and $D$ is a bounded domain with smooth boundary. The convex set $K$ is defincd by

$$
K=\left\{v \in H_{0}^{1}(D):|v(x)| \leq \operatorname{dist}(x, \partial D), x \in D\right\}
$$


where $\operatorname{dist}(x, \partial D)$ is the distance function to the boundary of $D$, and $H_{0}^{1}(D)$ is the Hilbert space of all functions with compact support in $D$ such that $v$ and $\|\nabla v\|^{2}$ belong to $L^{2}(D)$. This formulation and the physical interpretation of the torsion problem are discussed in the test problem collection of MINPACK-2 [1]. A finite element approximation of the torsion problem leads to

$$
q(v)=\frac{1}{2} \sum q_{i, j}^{L}(v)+\frac{1}{2} \sum q_{i, j}^{U}(v)-h_{x} h_{y} \sum w_{l}\left(z_{i, j}\right) v_{i, j},
$$

where

$$
\begin{aligned}
& q_{i, j}^{L}(v)=\mu_{i, j}\left\{\left(\frac{v_{i+1, j}-v_{i, j}}{h_{h x}}\right)^{2}+\left(\frac{v_{i, j+1}-v_{i, j}}{h_{h y}}\right)^{2}\right\}, \\
& q_{i, j}^{U}(v)=\lambda_{i, j}\left\{\left(\frac{v_{i-1, j}-v_{i, j}}{h_{h x}}\right)^{2}+\left(\frac{v_{i, j-1}-v_{i, j}}{h_{h y}}\right)^{2}\right\},
\end{aligned}
$$

and $\mu_{i, j}, \lambda_{i, j}, h_{x}$, and $h_{y}$ are constants.

Note that $q_{i, j}^{L}(v)$ and $q_{i, j}^{U}(v)$ are quadratics which depend only on $v_{i+1, j}, v_{i, j+1}, v_{i, j}$, and on $v_{i-1, j}, v_{i, j-1}, v_{i, j}$, respectively. The third contribution to $q(v)$, which is the linear part, depends only on $v_{i, j}$. So if we define

$$
\begin{array}{r}
S_{1}=\sum q_{i, j}^{L}(v) \\
S_{2}=\sum q_{i, j}^{U}(v) \\
S_{3}=\sum w_{l}\left(z_{i, j}\right) v_{i, j},
\end{array}
$$

then

$$
f(x)=\frac{1}{2} S_{1}+\frac{1}{2} S_{2}-h_{x} h_{y} S_{3}
$$

In the MINPACK-2 code for the torsion problem shown in Appendix A, LOOP1, LOOP2, and LOOP3 correspond to the computation of $S_{1}, S_{2}$, and $S_{3}$, respectively.

The torsion problem is a particular instance of a particular class of functions that arises often in optimization contexts, the so-called partially separable functions $[11,17,19]$. These are functions $f: \mathbb{R}^{n} \rightarrow \mathbf{R}$ which can be expressed as

$$
f(x)=\sum_{i=1}^{n b} a_{i} f_{i}(x) .
$$

Usually each $f_{i}$ depends on only a few (say, $n_{i}$ ) of the $x$ 's, and one can take advantage of this fact in computing the (sparse) Hessian of $f$.

ADIFOR (Automatic Differentiation of Fortran) is a source transiator that augments Fortran codes with statements for the computation of derivatives $[3,2]$. ADIFOR employs a mixed forward/reverse mode paradigm. The forward mode propagates derivatives of intermediate variables with respect to the input variables; the reverse mode propagates derivatives of the final values with respect to intermediate variables [14]. The forward mode follows the flow of execution of the original program, whereas the reverse mode of automatic differentiation requires the ability to access values generated in the execution of a program in reverse order, which is usually achieved by logging all values on a so-called tape, and then interpreting the tape in reverse order $[14,16,15]$. ADIFOR pioncered the use of the compile-time reverse mode where, insiead of logging values at run time, we apply the reverse mode at compile time, thereby eliminating the storage requirements and run-time overhead of the tape scheme.

In this paper, we are concerned with the efficient generation of derivative code through the reverse mode of automatic differentiation, and the efficient use of the generated derivative code for 


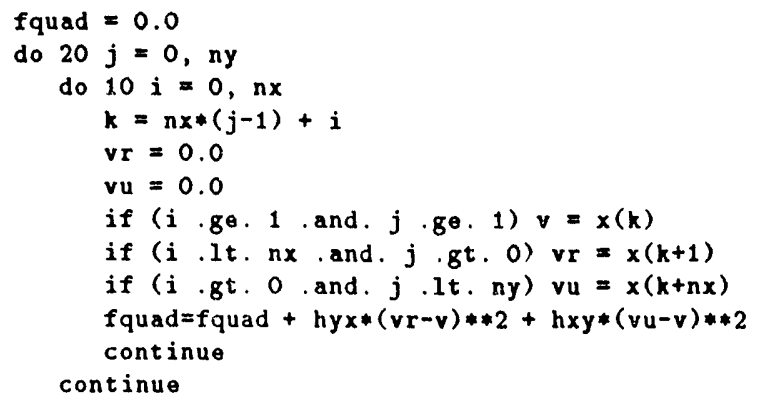

Figure 1: Code for LOOP1

computing gradients of partially separable functions. We use the torsion problem as a case study and explore how to improve the current ADIFOR-generated code and decrease the time and storage complexity of computing derivatives.

The paper is structured as follows. In the next section, we recall the key points about the method that is currently used in ADIFOR to generate derivatives. In Section 3, we then illustrate extensions of the compile-time reverse mode from basic blocks all the way to generating an adjoint code for the whole program. In Section 4, we explore the use of partial separability in computing derivatives. We present experimental results on Sparc-2 and IBM RS6000/550 workstations in Section 5.

\section{Current ADIFOR Strategy}

Automatic differentiation techniques rely on the fact that every function, no matter how complicated, is executed on a computer as a (potentially very long) sequence of elementary operations such as additions, multiplications, and elementary functions such as $\sin$ and $\cos$. By applying the chain rule

$$
\left.\frac{\partial}{\partial t} f(g(t))\right|_{t=t_{0}}=\left(\left.\frac{\partial}{\partial s} f(s)\right|_{s=g\left(t_{0}\right)}\right)\left(\left.\frac{\partial}{\partial t} g(t)\right|_{t=t_{0}}\right)
$$

over and over again to the composition of those elementary operations, one can compute derivative information of $f$ exactly and in a completely mechanical fashion [5]. ADIFOR transforms Fortran 77 programs using this approach.

To illustrate automatic differentiation with current ADIFOR, we differentiate the subroutine tor $f \mathrm{cn}$ for the torsion problem that maps an $n$-vector $\mathbf{x}$ into a scalar $\mathbf{f}$. The vector $\mathbf{x}$ contains the independent variables, and the scalar $f$ contains the dependent variable. The full code for torfen can be found in the appendix.

The first loop (LOOP1) is shown in Figure 1. It computes $S_{1}$, whose value is stored in fquad. Currently, ADIFOR generates the code shown in Figure 2 for computing $\frac{d f q u a d}{d \mathbf{X}}$. In accordance with the specification of ADIFOR (see [3]), $g \$ p$ denotes the actual length of the derivative objects in a call to derivative code. Since Fortran 77 does not allow dynamic memory allocation, derivative objects for local variables are statically allocated with leading dimension pmax. pmax is specified by the user when ADIFOR processes the Fortran code for torfen. A variable and its associated objects are treated in analogous manner; that is, if $\mathbf{x}$ is function parameter, so is $\mathbf{g} \$ \mathbf{x}$. Derivative objects corresponding to locally declared variables or variables in common blocks are declared as local variables or variables in common blocks. Given $\mathbf{x}$ and $\mathbf{g} \$ \mathbf{x}$, the derivative code comptites 


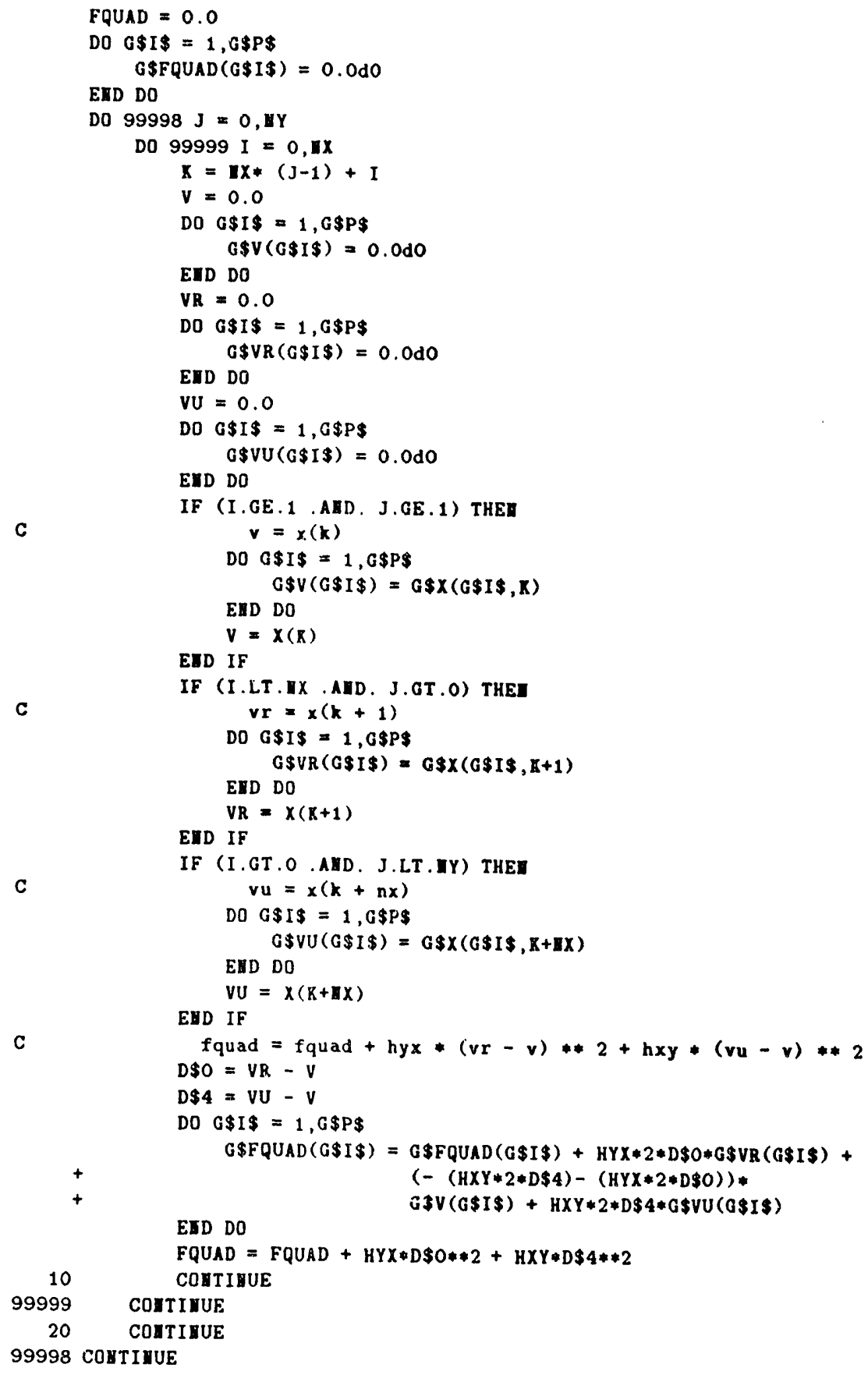

Figure 2: ADIFOR-generated Derivative Code for LOOP1 


$$
\operatorname{g} \$ \mathbf{q u a d}(1: \mathrm{g} \$ \mathrm{p})=\left(\left(\frac{d f q u a d}{d x}\right) g \$ x(1: g \$ p, 1: n)^{T}\right)^{T}
$$

In particular, if $\mathrm{g} \$ \mathrm{p}$ equals $n$ and $\mathrm{g} \$ \mathrm{x}$ is the $n \times n$ identity matrix, it computes the gradient of $f$ quad with respect to $\mathbf{x}$.

An active variable is one that is on the computational path from independent to dependent variables (sce [4]). Notice that in the ADIFOR-generated code, a loop of length $g \$ p$ is associated with every assignment staternent involving an active variable. Therefore the cost of floating-point opcrations can be approximated as $(g \$ p \times$ function evaluation). The storage reciuirement for ADIFOR-generated code is $(g \$ p \times$ number of active variables). We note two key points about the current ADIFOR:

- ADIFOR uses the forward mode overall to compute derivatives. That is, ADIFOR code maintains the derivatives of intermediate variables with respect to all input variables. So, for example, $g \$ v u=\frac{d v u}{d x}$.

- ADIFOR uses the reverse mode to preaccumulate "local" derivatives for assignments statements.

The reverse mode is best understood with an example. For example, in the torsion problem, we have the assignment

$$
\text { fquad }=\mathrm{hyx} *(\mathrm{vr}-\mathrm{v}) * * 2+\mathrm{hxy} *(\mathrm{vu}-\mathrm{v}) * * 2,
$$

where hxy and hyx are constants. The chain rule tells us that

$$
\nabla \mathrm{fquad}=\frac{\partial \text { fquad }}{\partial \mathrm{vr}} * \nabla \mathrm{vr}+\frac{\partial \text { fquad }}{\partial \mathrm{vu}} * \nabla \mathrm{vu}+\frac{\partial \text { fquad }}{\partial \mathrm{v}} * \nabla \mathrm{v}
$$

Ilence, if we know the "local" derivatives ( $\left.\frac{\partial \text { fquad }}{\partial v}, \frac{\partial \text { fquad }}{\partial v u}, \frac{\partial \text { fquad }}{\partial v r}\right)$ of fquad with respect to $v, v u$, and $v u$, we can easily compute $\nabla \mathbf{w}$, the derivatives of $\boldsymbol{w}$ with respect to $\mathbf{x}$. The "local" derivatives $\left(\frac{\partial \text { fquad }}{\partial \mathrm{v}}, \frac{\partial \text { fquad }}{\partial \mathrm{vu}}, \frac{\partial \text { fquad }}{\partial \mathrm{vr}}\right)$ can be computed efficiently by using the reverse mode of automatic differentiation. Here we maintain the derivative of the final result with respect to an intermediate quantity. These quantities are usually called adjoints. They measure the sensitivity of the final result with respect to some intermediate quantity. In the reverse mode, let tbar denote the adjoint object corresponding to $t$. The goal is for tbar to contain the derivative $\frac{\partial \text { fquad }}{\partial t}$. We know that wbar $=\frac{\partial \text { fquad }}{\partial \text { fquad }}=1.0$. We can compute ybar and zbar by applying the following simple rule to the statements executed in computing fquad, but in reverse order:

$$
\begin{aligned}
\text { if } s=f(t), & \text { then tbar }+=\text { sbar } *(d f / d t) \\
\text { if } s=f(t, u), & \text { then tbar } t=s \text { bar } *(d f / d t) \\
\text { ubar } t & =s \text { sar } *(d f / d u)
\end{aligned}
$$

Using this simple recipe (see $[14,20]$ ), we generate the code shown in Figure 3 for computing vubar, vrbar, and vbar. One can easily convince oneself that

$$
\begin{aligned}
& \text { vubar }=2 * \text { hxy } *(v x-v) \\
& \text { vrbar }=2 * \text { hyx } *(v r-v) \\
& \text { vbar }=-2 * h x y *(v u-v)-2 * \text { hy } x *(v r-v)
\end{aligned}
$$

so that we have in fact computed the correct "local" derivatives. The code shown in Figure 2 has been generated by applying this same technique to all other assignments statements involving active variables and by optimizing the resulting code by removing additions with 0 and multiplications

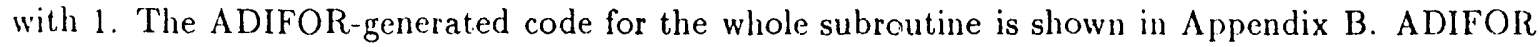
is currently not consistent about pulling loop invariant subexpressions out of the loop, but will do so reliably in the future. 


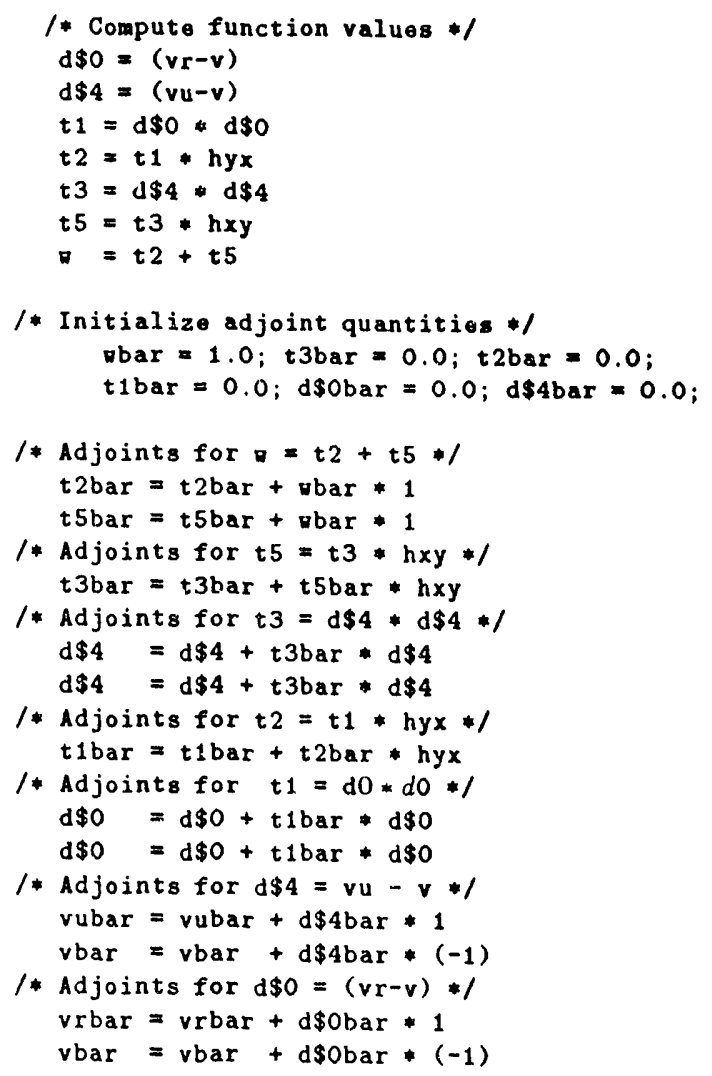

Figure 3: Unoptimized Reverse Mode Computation 


\section{Extending the Scope of the Compile-Time Reverse Mode}

In this section, we explore extensions of the compile-time reverse mode to

- a sequence of assignment statements,

- a nested loop, and

- the whole program.

A closer look at the current ADIFOR-generated code in the preceding sections reveals a substantial time and space overhead associated with the computations of auxiliary gradients such as $g \$ v$, $g \$ v u$, and $g \$ v r$. In this section, we explore different ways for improving the overall computation of the gradient by extending the scope of the reverse mode.

\subsection{Case 1: Reverse Mode for Basic Blocks inside the Loop}

In the program for the torsion problem, there are three loops: two for the computation of the quadratic part of the function and one for the computation of the linear part. Consider, for example, LOOP1. Each loop iteration can be viewed as a mapping

$$
\left[x(k), x(k+1), x(k+n x), \text { fquad }_{0 l d}\right] \longmapsto \text { fquad }_{n e w} .
$$

We use the notation fquad $_{\circ \mathrm{dd}}$ and fquad $_{\text {new }}$ to distinguish between the original and updated value of the variable fquad. Hence, if we know

$$
\frac{\partial \text { fquad }_{\text {new }}}{\partial \mathbf{x}(\mathrm{k})}, \frac{\partial \text { fquad }_{\text {new }}}{\partial \mathbf{x}(\mathrm{k}+1)}, \frac{\partial \text { fquad }_{\text {new }}}{\partial \mathbf{x}(\mathrm{k}+\mathrm{nx})} \text {, and } \frac{\partial \text { fquad }_{\text {new }}}{\partial \text { fquad }_{\text {old }}},
$$

then we can update $\nabla$ fquad as follows:

$$
\begin{aligned}
\nabla \text { fquad }= & \frac{\partial \text { fquad }_{\text {new }}}{\partial \mathbf{x}(\mathrm{k})} \nabla \text { fquad }+\frac{\partial \text { fquad }_{\text {new }}}{\partial \mathbf{x}(\mathrm{k}+1)} \nabla \mathbf{x}(\mathrm{k}+1) \\
& +\frac{\partial \text { fquad }_{\text {new }}}{\partial \mathbf{x}(\mathrm{k}+\mathrm{nx})} \nabla \mathbf{x}(\mathrm{k}+\mathrm{nx})+\frac{\partial \text { fquad }_{\mathrm{n}} \mathrm{w}}{\partial \text { quad }_{\text {old }}} \nabla \text { fquad. }
\end{aligned}
$$

The derivatives in the equation (2) can easily be computed by applying the reverse mode to the loop body. The resulting code is shown in Figure 4 . Note that each variable is assigned only once in each loop iteration. If this had not been the case, we would have had to save the sequence of values of variables that are overwritten by allocating some extra temporary variables. This extension of the scope of the reverse mode saved us 3 derivative vectors $g \$ v, g \$ v r$, and $g \$ v u$, and decreased the number of derivative vector operations from 10 to 4 .

In general, we can apply this technique in a straightforward fashion to any piece of code that has only one entry and exit point and does not contain subroutine or function calls or loops. We call such a piece of code a basic block. We may have to introduce some temporaries to make sure that each variable is assigned only once (i.e., represents a unique value) in a basic block, but this requires at most as many scalar temporaries as there are lines of code, an insignificant increase of storage. The savings achieved by this technique depend on the particular code at hand, but, in general, will be the more pronounced the more statements a basic block contains. The code that results from applying this technique to the whole subroutine is shown in Appendix C.

\subsection{Case 2: Reverse Mode for the Whole Loop}

In order to expand the scope of the compile-time reverse mode, the special structure of the torsion problem is important Defining

$$
t_{\mathrm{k}}:=-\mathrm{hyx} *(\mathrm{vr}-\mathrm{v}) * * 2+\mathrm{hxy} *(\mathrm{vu}-\mathrm{v}) * * 2
$$




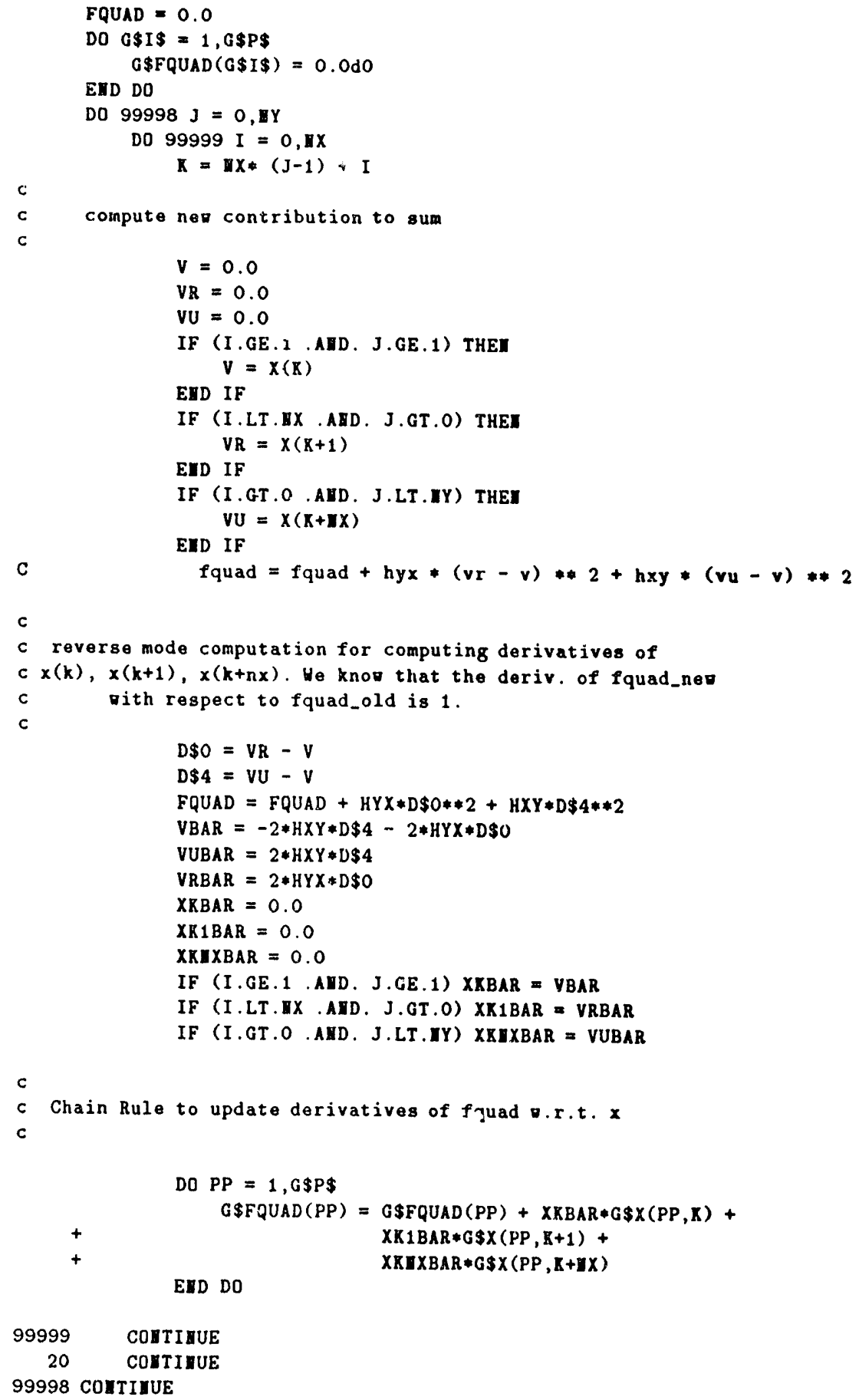

Figure 4: Reverse Mode for Basic Block in LOOP1 
to be the value computed in loop iteration $\mathbf{k}$ to upgrade Iquad, we can express

$$
\text { Iquad }=\sum_{k=1}^{(n x+1)(n y+1)} t_{k}
$$

Since $v, v u$, and vr are defined in terms of $z(k+1), x(k+n x)$, and $x(k), t_{k}$ is a function of these values, that is,

$$
t_{k}=t_{k}(x(k+1), x(k+n x), x(k)) .
$$

Since no entry of $x$ is overwritten in any of the loop iterations, $t_{k}$ and $t_{1}$ do not depend on each other for $k \neq 1$, and we can compute the sum (3) in any order. In compiler terms, there are no loop-carried dependencies and this loop is a parallel loop.

Remember that the reverse mode implicitly assumes that we are able to trace the values computed during some computation in the reverse order. Hence, a loop that is not parallel would require us to sam some intermediate values. However, for a parallel loop, it is sufficient simply to generate the :iverse mode code for the loup body. But this is exactly what we already did in the preceding section, where we computed

$$
\frac{\partial t_{k}}{\partial x(k+1)}, \frac{\partial t_{k}}{\partial x(k)}, \frac{\partial t_{k}}{\partial x(k+n x)} .
$$

Now, since $t_{l}$ and $t_{k}$ do not depend on each other for $i \neq k$, the associativity of addition allows us to compute

$$
\frac{d \text { quad }}{a x(j)}=\frac{\partial t_{j-1}}{\partial x(j)}+\frac{\partial t_{j-n x}}{\partial x(j)}+\frac{\partial t_{j}}{\partial x(j)}
$$

in a piecemeal fashion, as each of the iterations $j, j-1$, and $j-n \times$ contributes to $\frac{\partial \mathfrak{q u a d}}{\partial \mathbf{x}(\mathrm{j})}$. 'The resu: ny code is shown in Figure 5 . The xbar vector contains $\frac{d f q u a d}{d x}$ and components $k+1, k$, and $k+r x$ updated in iteration $k$. After the loop, we apply the chain rule to compute

$$
\nabla \mathfrak{f q u a d}=\frac{d \mathfrak{f q u a d}}{d x} \cdot \nabla \mathbf{x} .
$$

This matrix-vector multiplication is performed using the BLAS routine DGEMV [13].

To summarize, we exploited the fact that

- loop iterations do not depend on each other, and

- the result of each loop enters into the dependent variable (Iquad) in an additive fashion.

1 his allowed us to generate reverse mode code for the whole loop by simply generating reverse mode cc de for the loop body, and the forward mode propagation of the global derivatives could be moved ou'side of the loop.

Compar $\epsilon$ with the code in the previous section, we now have a multiplication of an $g \$ p \times(n x+1)$ $(n y+1)$ matrix by a vector outside the loop instead of $(n x+1)(n y+1)$ multiplications of an $g \$ p \times 4$ inatrix by a vector multiplication inside a loop that is executed $(n x+1)(n y+1)$ times, requiring roughly one-fourth the number of operations. Applying this technique to the whole subroutine resu ts in the code shown in Aprendix D.

\subsection{Case 3: The Full Reverse Mode}

So far we exploited only the particular structure of the code in LOOP1, LOOP2, and LOOP3. On ihe other hand, $f(x)$ is the sum of the contributions computed in LOOP1, LOOP2, LOOP3, atud, in addition to being parallel loops themselves, these loops do not depend on each other. So, instead of computing 


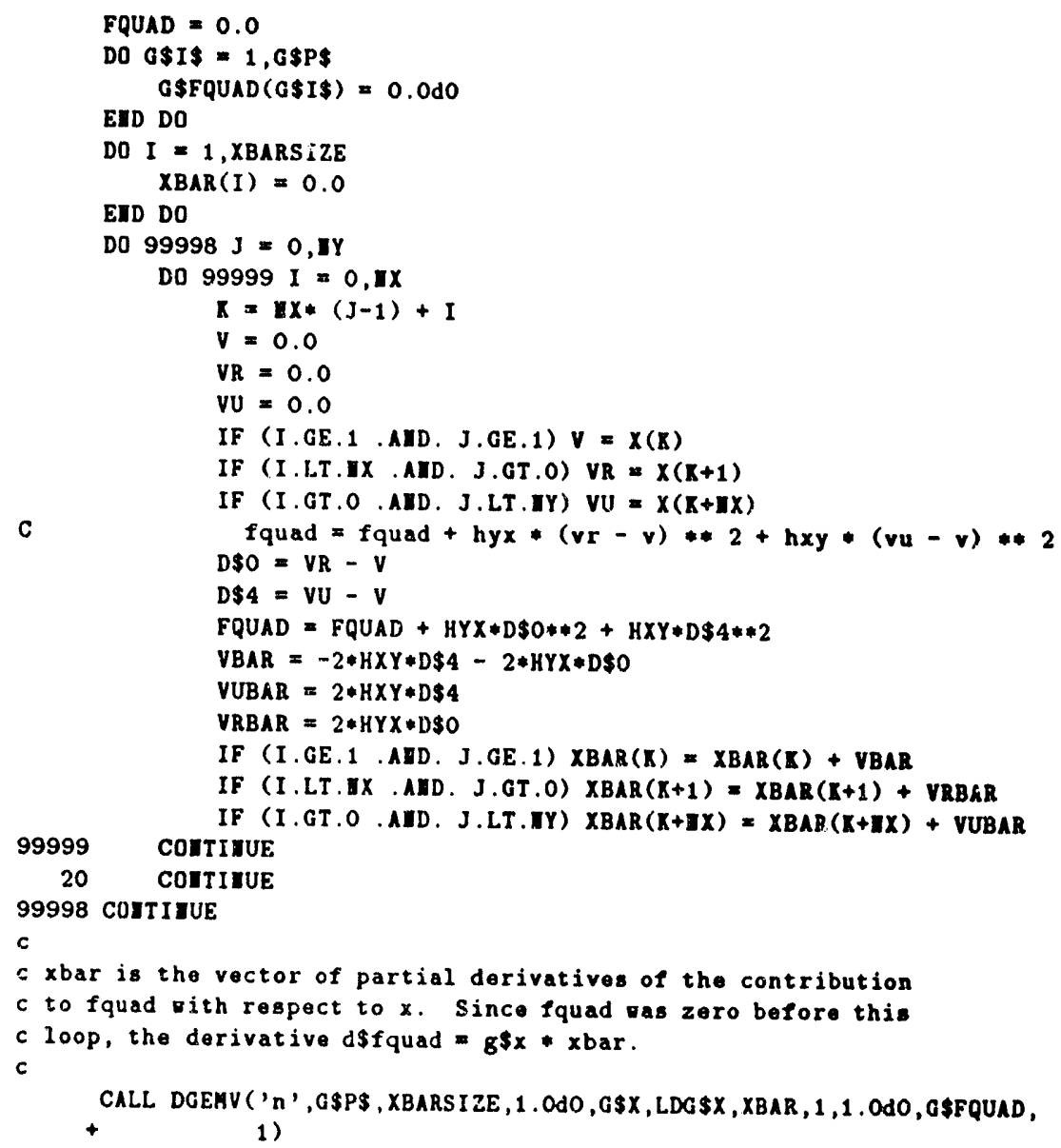

Figur 5i Reverse Mode for the Whole Loop 


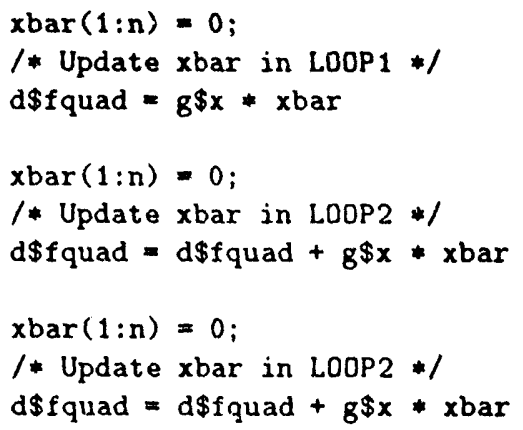

we could simply keep on updating xbar in LOOP1, LOOP2, and LOOP3 and perform compute $\mathrm{d} f$ quad $=g \mathrm{x} * \mathbf{x b a r}$ once at the end. This is possible since none of these loops updates the vector $x$, and hence $g \$ x$ remains ur,changed. But we can go even further: Since in the forward mode, $g \$ x$ is initialized to the identity, we can eliminate the final multiplication $\mathrm{g} \$ \mathrm{q}$ quad $=g \$ x * x$ bar and simply ussign return xbar. In this fashion, we have generated adjoint code for the whole subroutine, and the code for computing the gradient does not contain any vector operations.

It is important to note that we were able to do the full implementation of the reverse mode because

- each of the three loops is a parallel loop,

- the three loops do not depend on each other,

- the contribution computed inside each loop enters in the final result in an additive fashion, and

- the results of each of the three loops are added to achieve the final result.

The resulting reverse mode code for the torsion problem is shown in Appendix E. While we did not decrease the storage requirement any further compared with the preceding section, we saved another three loops of size $\mathbf{g} \$ \mathrm{p} n \boldsymbol{n} \mathbf{n y}$, and the run time of this program no longer depends on $\mathbf{g} \$ \mathrm{p}$.

\section{Exploring Partial Separability}

As was mentioned in the introduction, the torsion problem is a partially separable function $f: \mathbf{R}^{n} \rightarrow \mathbf{R}$, in that it can be expressed as

$$
f(x)=\sum_{i=1}^{n b} f_{i}(x)
$$

This structure can also be used advantageously in computing the (usually dense) gradient $\nabla f$ of $f$ (sce [9]). Assume that the code for computation of $f$ looks as follows:

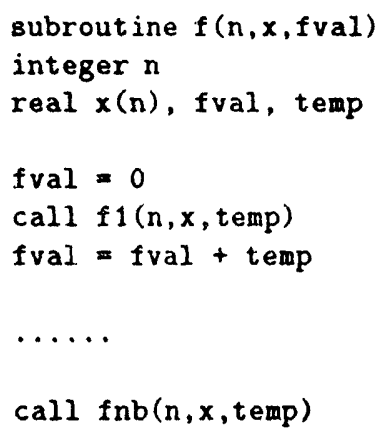




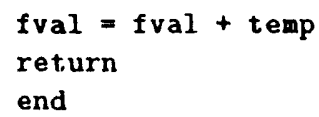

If we submit $f$ to ADIFOR, it generates

subroutine $g \$ n(n, x, g \$ x, l d g \$ x, f v a l, g \$ f v a l, l d g \$ f v a l)$.

To compute $\nabla f$, the first (and only) row of the Jacobian of $f$, we set $g \$ \mathrm{p}=n$ and initialize $g \$ x$ to a $n \times n$ identity matrix. Hence, in current ADIFOR, the cost of computing $\nabla f$ is of the order of $n$ times the function evaluation.

As an alternative, we realize that with $f: \mathbf{R}^{n} \rightarrow \mathbf{R}^{n b}$ defined as

$$
\boldsymbol{g}=\left(\begin{array}{c}
f_{1} \\
\vdots \\
f_{n b}
\end{array}\right),
$$

we have the identities

$$
f(x)=e^{T} g(x), \text { and hence } \nabla f(x)=e^{T} J_{g},
$$

where $e$ is the vector of all ones, and $J_{g}$ is the Jacobian of $g$. However, if most of the component functions $f_{i}$ depend only on a few parameters $x_{j}$, the Jacobian of $g$ is sparse, and this fact can be exploited advantageously.

The idea is best understood with an example. Assume that we have a function

$$
F=\left(\begin{array}{c}
f_{1} \\
f_{2} \\
f_{3} \\
f_{4} \\
f_{5}
\end{array}\right): x \in \mathbf{R}^{4} \mapsto y \in \mathbf{R}^{5}
$$

whose Jacobian $J$ has the following structure (symbols denote nonzeros, and zeros are not shown):

$$
J=\left(\begin{array}{cccc}
\bigcirc & & & \\
\bigcirc & & & \diamond \\
& \triangle & & \diamond \\
& \triangle & \square & \\
& \triangle & \square &
\end{array}\right) .
$$

That is, the function $f_{1}$ depends only on $x_{1}, f_{2}$ depends only on $x_{1}$ and $x_{4}$, and so on. The key idea in computing sparse Jacobians is to identify so-called structurally orthogonal columns $j_{i}$ of $J$ (see [10]), that is, columns whose inner product is always zero, independent of the numerical values of their nonzero entries. In our example, columns 1 and 2 are structurally orthogonal, and so are columns 3 and 4 . This means that the set of functions that depend nontrivially on $x_{1}$, namely $\left\{f_{1}, f_{2}\right\}$, and the set of functions that depend nontrivially on $x_{2}$, namely $\left\{f_{3}, f_{4}, f_{5}\right\}$, are disjoint. Because of the graph-coloring approaches that are used to reveal this structure, one usually associates a "color" with every set of structurally orthogonal columns.

To exploit this sparsity structure, we recall that ADIFOR (ignoring transposes) computes $J \cdot S$, where $S$ is a matrix with $g \$$ p columns. For our example, setting $S=I_{4 \times 4}$ will give us $J$ at roughly four times the cost of evaliuating $f$, but if we exploit the structural orthogonality and set

$$
S=\left(\begin{array}{ll}
1 & 0 \\
1 & 0 \\
0 & 1 \\
0 & 1
\end{array}\right)
$$


the running time for the ADIFOR code is roughly halved. The ADIFOR-generated code remains unchanged.

This idea can readily be applied to the torsion problem. By storing the contribution of iteration $k$ to Iquad in the $k$-th clement of separate vectors FQ, FQQ, and FP (for the LOOP1, LOOP2, and LOOP3, respectively), the derivative of fquad is the sum of the row sums of the Jacobians of FQ, FQQ, and FP.

For example, the code for the loop corresponding to $F Q$ is

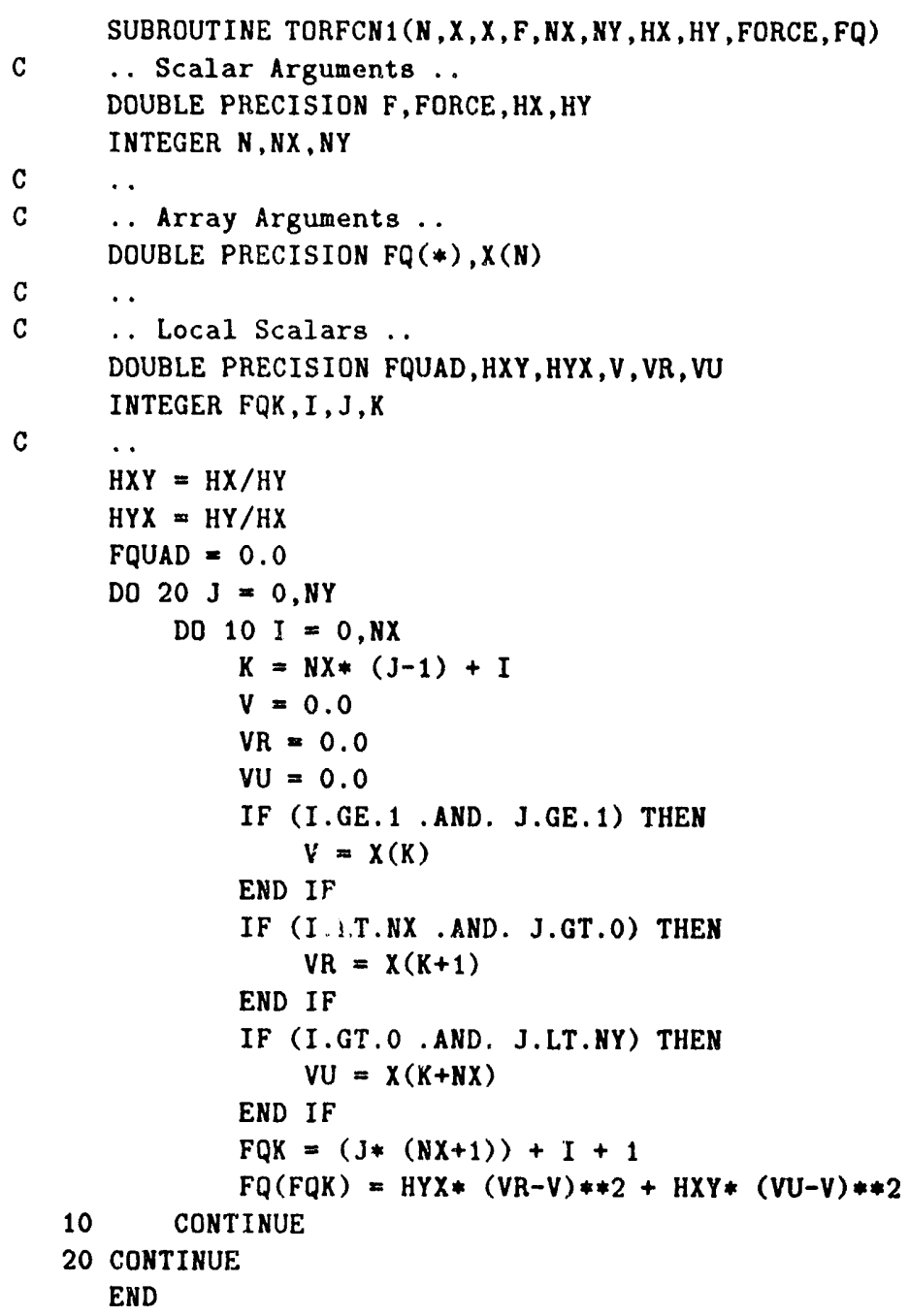

The only change (compared with the corresponding code fragment in Appendix A) is that we replaced the accumulation of fquad by an assignment to FQ. Subroutines torfen 2 and torf $\mathrm{cn} 3$ to compute FQQ and FP, respectively, are generated in the same fashion. For these codes, ADIFOR then generates the derivative codes shown in Appendix F.

For $n=40$, the structures of $\frac{d F Q}{d x}$ and $\frac{d F Q Q}{d x}$ are shown in Figure 6 , and $\frac{d F P}{d x}$ is diagonal. The Jacobian for $F Q$ and FQQ can be grouped into three sets of structurally orthogonal columns, independent of the size of the problem. And in the case of the function FP. the Jacobian can be compressed into only one column.

Exploiting this structure, we can now initialize the gradient vector as follows: 


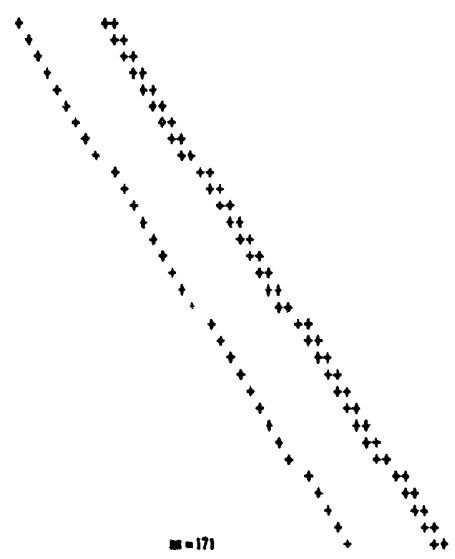

g\$FQ

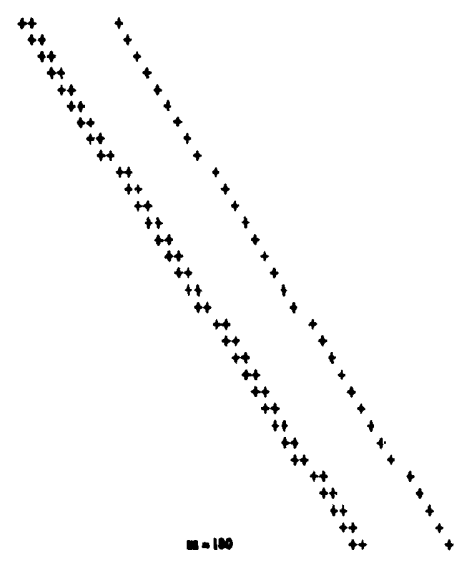

g\$FQQ

Figure 6: Sparsity Structures of Component Jacobians

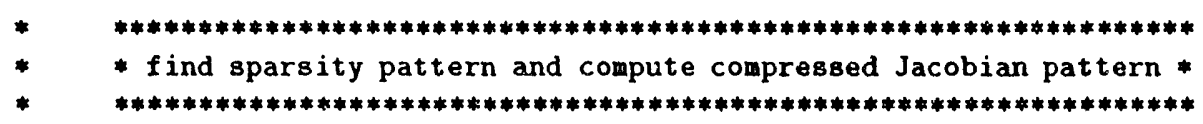

CALL SPARSITY ( $N, X, F, H X, N Y, H X, H Y$, INDROHQ, INDCOLQ, NNZQ, INDROWQQ, INDCOLQQ, NNZQQ)

DO $I=1, N N Z Q$

$\operatorname{INDRONQS}(\mathrm{I})=\operatorname{INDROWQ}(\mathrm{I})$

$\operatorname{INDCOLQS}(I)=\operatorname{INDCOLQ}(I)$

INDROWQQS (I) $=\operatorname{INDROWQQ(I)~}$

$\operatorname{IHDCOLQQS}(I)=\operatorname{INDCOLQQ}(\mathrm{I})$

END DO

CALL DSM ( (NY+1)* (NX+1), N, NNZQ, INDROWQ, INDCOLQ, NGRPQ, MAXGRPG, MINGRPQ, INFO, IPNTRQ, JPNTRQ, IWA , LIWA)

CALL DSM ( $(N X+1) *(N Y+1), N$, NKZQQ, INDROWQQ, INDCOLQQ , NGRPQQ, MAXGRPQQ, MINGRPQQ, INFO, IPNTRQQ, JPNTRQQ, IHA , LIWA)

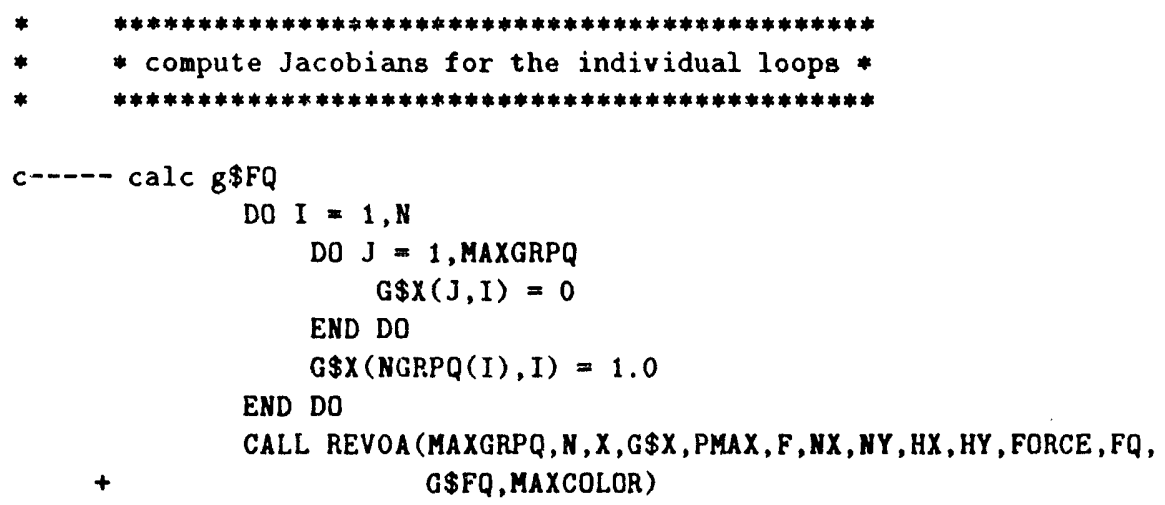




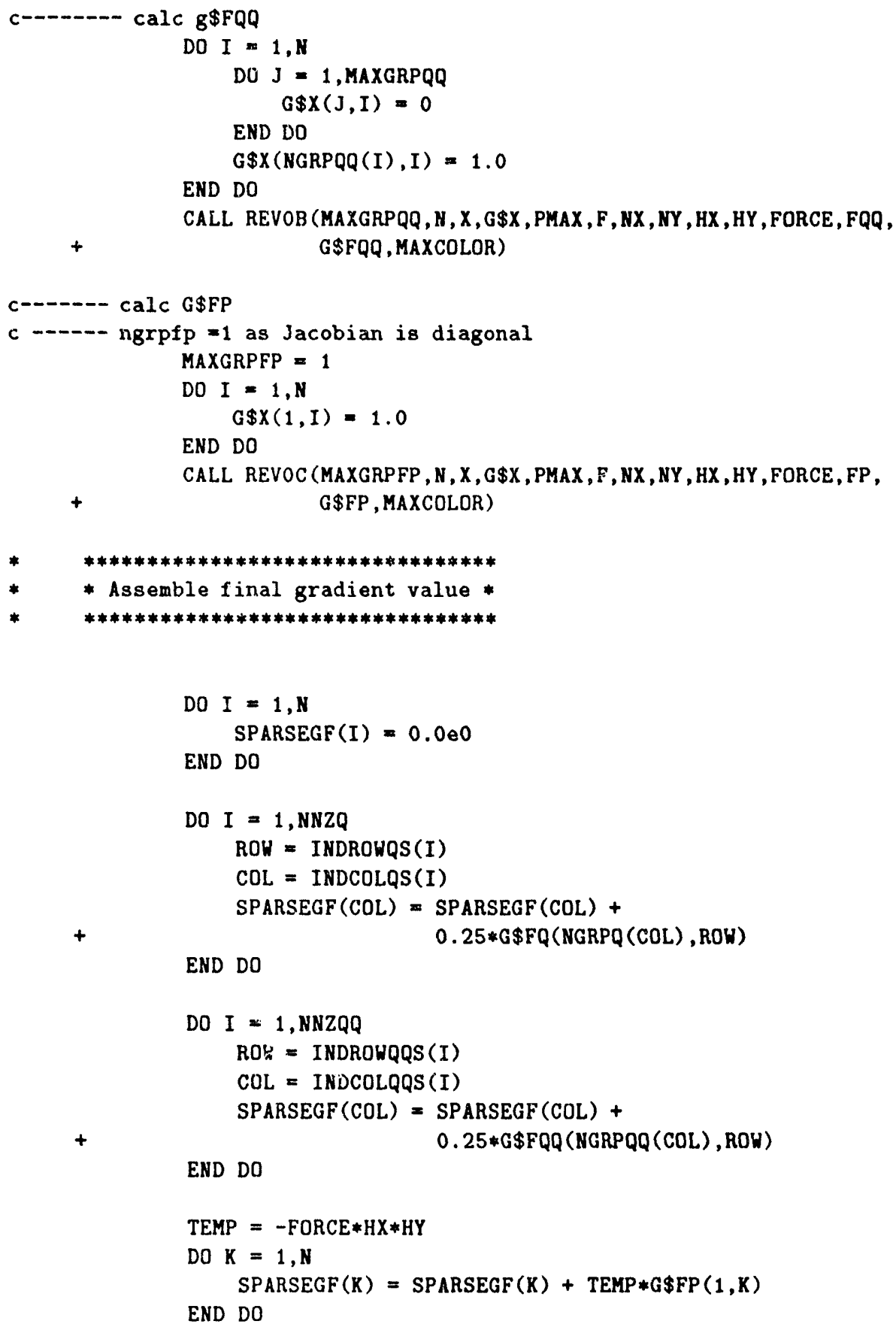

After we have initialized some arrays determining the sparsity pattern of the Jacobian, we call the MINPACK subroutine DSM [9] to determine the proper coloring for the Jacobians of FQ and FQQ. Ilaving thus determined NGRPQ( $i$ ), the "color" of column $i$ and MAXGRPQ, the number of colors for the Jacobian of $F Q$, we initialize $\mathbf{g} \$ \mathbf{x}$ and calls revOa (a renamed version of the ADIFOR-generated subroutine for torf $\mathrm{cn} 1$ ) to compute the compressed Jacobian of FQ. The same idea is applied to compute $\mathbf{\$} \$ \mathrm{FQ}$ and $\mathrm{g} \$ F P$. Lastly, the derivative values of the subfunctions are all added into a sparse vector, without ever expanding the compressed component Jacobians, as shown below. For the Jacobian of FQ, the index arrays INDROWQS and INDCOLQS indicate the row and column index of 
nonzero entries, and the NGRPQ array indicates the group (corresponding to one particular color) of a certain column. The Jacobian of FQQ is dealt with accordingly. The uncompression of the Jacobian of FQ is trivial, since it was diagonal - we just add the $i$-th diagonal entry (properly scaled) to the $i$-th entry of the gradient accumulation vector SPARSEGF. The MINPAC $K$ documentation continins details on the particular data structures used to represent the sparse derivative matrices.

We note that we could of course apply the idea of the "basic block reverse mode" to generati improved derivative code for torfen1, etc. This code is shown in Appendix ( $i$. We would expect much less spectacular savings in this case, since the length of the derivative objects was not more than three for our sparse Jacobians (whereas it was $n$ when we did not exploit partial separability).

\section{Experimental Results}

We tested the performance of our various derivative codes on a Sun Sparcstation iPX with 48 Mbytes of memory and an IBM RS6000/550 with 128 Mbytes of memory. We computed gradients for $n=10 * 10,20 * 20, \ldots, 100 * 100$. For the alternatives described in Sections 2, 3.1, and 3.2, we computed gradients in slices of 10 elements (i.e., the gradient was computed by calling the derivative code $\lceil n / 10\rceil$ times). Figure 7 shows the ratio of the run time of a gradient to a function evaluation obtained for these derivative codes. As expected, the run time is linear in $n$, but the slope decreases as we expand the scope of the reverse mode.

In Figure 8 we show the ratio of the run time of a gradient to a function evaluation obtained by the full reverse mode (Section 3.3) and by exploiting the partial separability of the torsion problem. 'These graphs also show the run time of the handcoded derivative subroutine supplied in the MINPA(' $\mathrm{K}-2$ test suite. We see that by exploiting partial separability, we can achieve very good performance for computing the gradient of the torsion problem. This is particularly noteworthy as we do not need to know anything more about the structure of the problem than that it is partially separable. In contrast. intimate knowledge of the code is required to develop the full reverse modr and the handcoded versions.

\section{Acknowledgments}

We thank Alan (iarle, (ieorge Corliss, and Andreas Griewank for their comments. We are especially grateful to (ieorge ('orliss for his thoughtful and thorough comments on an earlier (lraft of this paper.

\section{References}

[1] Brett Averick, Richard (i. Carter, and Jorge J. Moré. The MINPACK-2 test problem collection (preliminary version). Technical Report ANL/MCS-TM-150, Mathematics and computer Sicience Division, Argonne National Laboratory, Argonne, Ill., 1991.

[2] Christian Bischof, Alan ('arle, George Corliss, and Andreas Ciriewank. ADIFOR: Automatic differentiation in a source translator environment. Preprint MCS P288-0192, Mathematics and Computer Science I)ivision, Argonne National Laboratory, January 1992. ADIFOR Working Note \#5. Accepted for publication in Proceedings of International S'ymposium on Symbolic and Algebraic Computilion.

[3] Christian Bischof, Alan Carle, George Corliss, Andreas Griewank, and Paul Hovland. ADIFOR: (ienerating derivative codes from Fortran programs. ADIFOR Working Note \#1, MCS P2633 0991, Mathematics and ('omputer Science Division, Argonne National Laboratory, 1991. To appear in Sicientifir Programming.

[4] Christian Bischof, Alan Carle, ( George Corliss, Andreas Griewank, and Paul Hovland. ADIF()R: Fortran source translation for efficient derivatives. Preprint MCS-P278-1291, Mathematics 
and Computer Science Division, Argonne National Laboratory, Argonne, Ill., December 1991. ADIFOR Working Note \# 4.

[5] Christian Bischof and Paul Hovland. Using ADIFOR to compute dense and sparse Jacobians. Technical Report ANL/MCS-TM-158, Mathematics and Computer Science Division, Argonne National Laboratory, Argonne, Ill., October 1991. ADIFOR Working Note \# 2.

[6] Kathy E. Brenan, Stephen L. Campbell, and Linda R. Petzold. Numerical Solution of InitialValue Problems in Differential-Algebraic Equations. North-Holland, New York, 1989:

[7] John C. Butcher. The Numerical Analysis of Ordinary Differential Equations (Runge-Kutta and General Linear Methods). John Wiley and Sons, New York, 1987.

[8] George F. Carrier and Carl E. Pearson. Partial Differential Equations. Academic Press, San Diego, California, 1988.

[9] T. F. Coleman, B. S. Garbow, and J. J. Moré. Software for estimating sparse Jacobian matrices. ACM Trans. Math. Software, 10:329-345, 1984.

[10] T. F. Coleman and J. J. Moré. Estimation of sparse Jacobian matrices and graph coloring problems. SIAM Journal on Numerical Analysis, 20:187-209, 1984.

[11] S. D. Conte and Carl de Boor. Elementary Numerical Analysis. McGraw-Hill, New York, 1980.

[12] John Dennis and R. Schnabel. Numerical Methods for Unconstrained Optimization and Nonlinear Equations. Prentice-Hall, Englewood Cliffs, N.J., 1983.

[13] Jack J. Dongarra, Jeremy Du Croz, Sven Hammarling, and Richard J. Hanson. An extended set of Fortran Basic Linear Algebra Subprograms. ACM Transactions on Mathematical Software, 14(1):1-17, 1988 .

[14] Andreas Griewank. On automatic differentiation. In Mathematical Programming: Recent Developments and Applications, pages 83-108, Amsterdam, 1989. Kluwer Academic Publishers.

[15] Andreas Griewank. Achieving logarithmic growth of temporal and spatial complexity in reverse automatic differentiation. Optimzzation Methods $\mathcal{B}$ Software, 1(1):35-54, 1992.

[16] Andreas Griewank, David Juedes, and Jay Srinivasan. ADOL-C, a package for the automatic differentiation of algorithms written in C/C++. Preprint MCS-P180-1190, Mathematics and Computer Science Division, Argonne National Laboratory, Argonne, Ill., November 1990.

[17] Andreas Griewank and Philippe L. Toint. Partitioned variable metric updates for large structured optimization problems. Numerische Mathematik, 39:119-137, 1982.

[18] E. Hairer and G. Wanner. Solving Ordinary Differential Equations II (Stiff and DifferentialAlgebrazc Problems), volume 14 of Springer Series in Computational Mathematics. SpringerVerlag, New York, 1991.

[19] Jorge J. Moré. On the performance of algorithms for large-scale bound constrained problems. In T. F. Coleman and Y. Li, editors, Large-Scale Numerical Optimization, pages $32-45$. SIAM, 1991.

[20] Louis B. Rall. Automatic Differentiation: Techniques and Applications, volume 120 of Lecture Noles in Computer Science. Springer-Verlag, Berlin, 1981.

[21] Erich Zauderer. Partial Differential Equations of Applied Mathematics. John Wiley \& Sons, Somerset, N.J., 1989. 

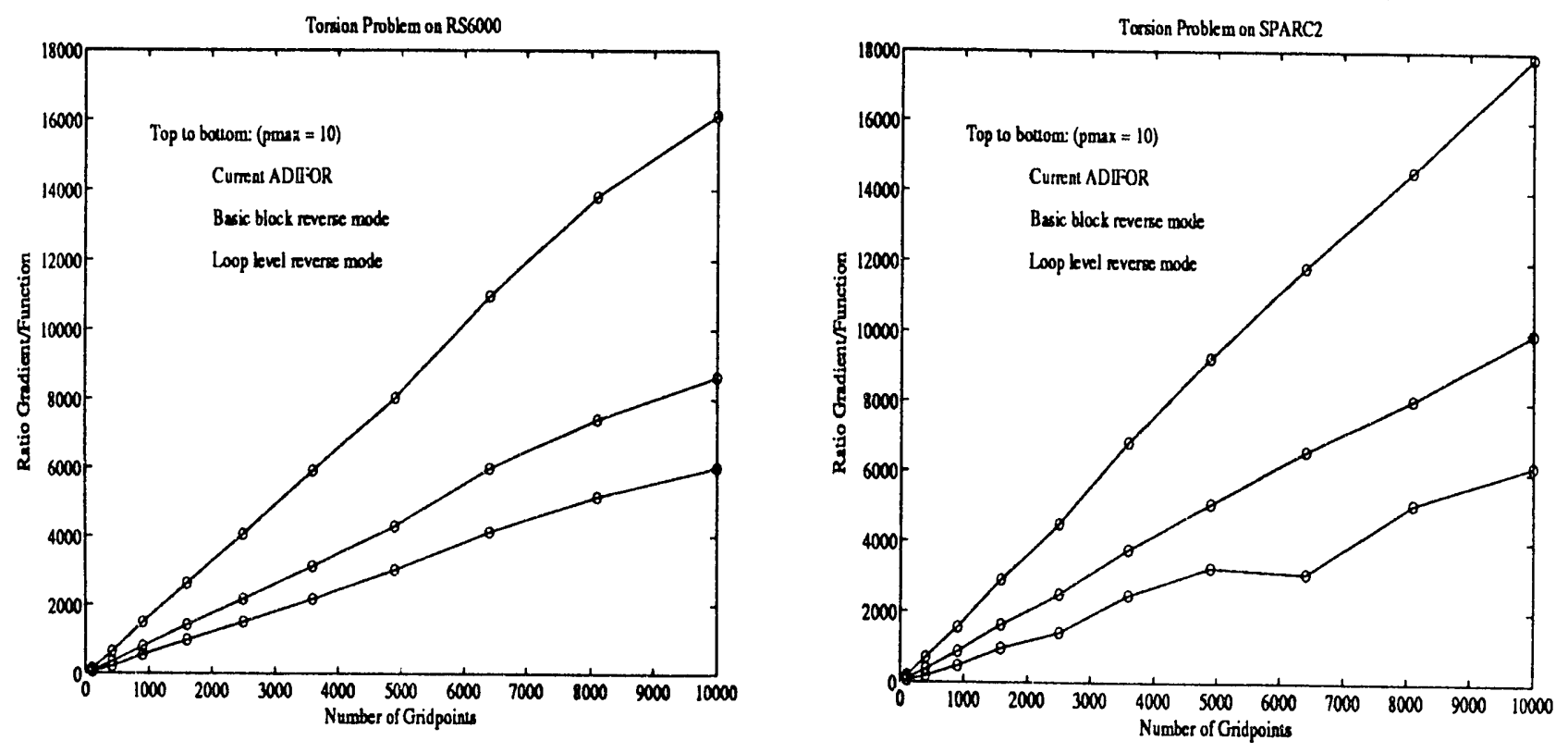

Figure 7: Performance of Current ADIFOR Code and Enhanced Versions
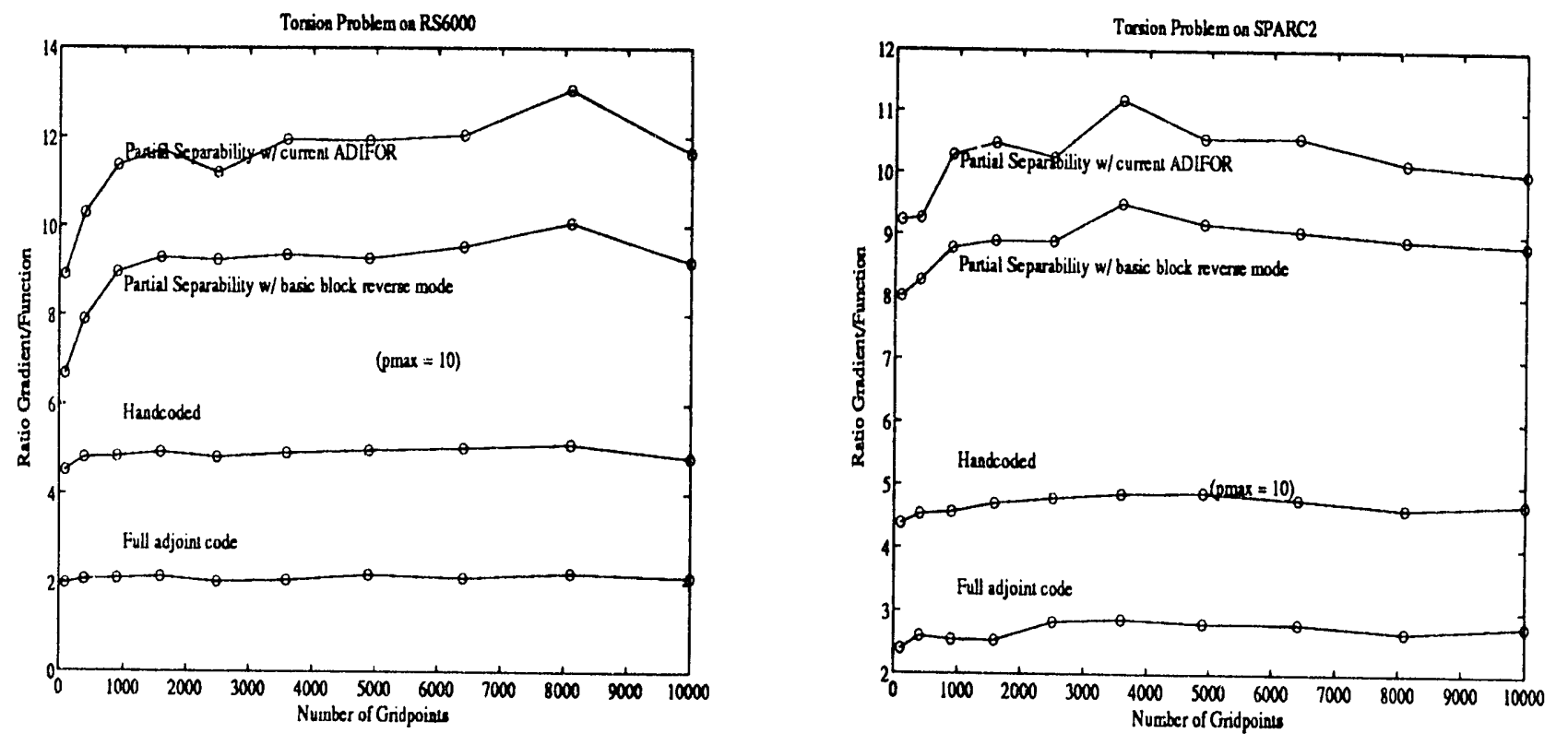

Figure 8: Exploiting Partial Separability, the Full Reverse Mode and IIandcoded Derivatives 
APPENDICES: Code Listings for the Torsion Problem

A Minpack-2 Code for the Torsion Problem

SUBROUTINE TORFCN ( $N, X, F, N X, N Y, H X, H Y, F O R C E)$

c This subroutine computes the function of the torsion problem.

c The spacing parameters $h x$ and hy are for a rectangle with

c nx points on the $x$-axis and ny points on the $y$-axis

C .. Scalar Arguments .. DOUBLE PRECISION F, FORCE, HX,HY INTEGER $\mathrm{N}, \mathrm{NX}, \mathrm{NY}$

C $\quad$.

C .. Array Arguments .. DOUBLE PRECISION $X(\mathrm{~N})$

C $\quad$.

C .. Local Scalars .. DOUBLE PRECISION FLIN, FQUAD, HXY, HYX, V, VD, VL, VR, VU INTEGER I, J,K

C

$H X Y=H X / H Y$

$H Y X=H Y / H X$

c Computation of the quadratic part of the function.

c LOOP 1 :

FQUAD $=0.0$

DO $20 \mathrm{~J}=0, \mathrm{NY}$

DO $10 I=0, N X$

$K=N X *(J-1)+I$

$V=0.0$

$\mathrm{VR}=0.0$

$\mathrm{VU}=0.0$

IF (I.GE. 1 .AND. J.GE. 1 ) $V=X(K)$

IF (I.LT.NX .AND. J.GT.0) VR $=X(K+1)$

IF (I.GT.0 .AND. J.LT.NY) VU $=X(K+N X)$

FQUAD $=$ FQUAD + HYX*(VR-V $) * * 2+H X Y *(V U-V) * * 2$

10 CONTINUE

20 CONTINUE

c

c LOOP2:

c

DO $40 \mathrm{~J}=1, \mathrm{NY}+1$

DO $30 I=1, N X+1$

$K=N X *(J-1)+I$

$v=0.0$

$V L=0.0$

$\mathrm{VD}=0.0$

IF (I.LE.NX .AND. J.LE.NY) $V=X(K)$

IF (I.GT. 1 .AND. J.LE.NY) VL $=X(K-1)$

IF (I.LE.NX .AND. J,GT.1) VD $=X(K-N X)$

FQUAD $=$ FQUAD + HYX* $(V L-V) * * 2+H X Y *(V D-V) * * 2$ 
30 CONTINUE

40 CONTINUE

c Computation of the linear part of the function.

C LOOP 3:

FLIN $=0.0$

DO $50 \mathrm{~K}=1, \mathrm{NX} * \mathrm{HY}$

FLIN $=$ FLIN + X(K)

50 CONTINUE

$F=0.25 *$ FQUAD - FORCE $* H X * H Y * F L I N$

END 
B Current ADIFOR Code for Torsion Problem

SUBROUTINE REVO(G\$P\$, $\boldsymbol{N}, X, G \$ X, L D G \$ X, F, G \$ F, L D G \$ F, N X, N Y, H X, H Y, F O R C E)$

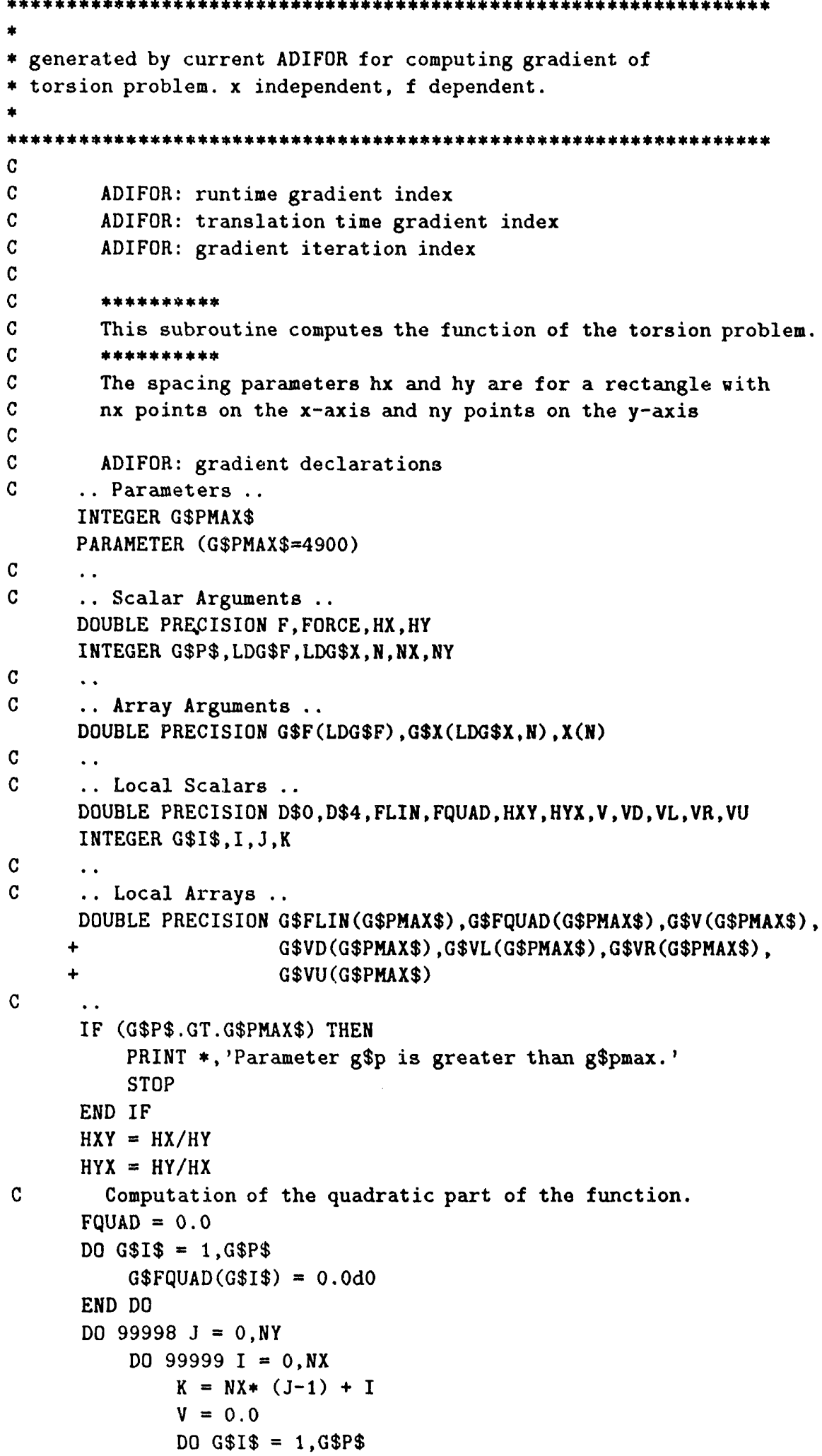


END DO

$\operatorname{GSV}(G \$ S)=0.0 \mathrm{~d} 0$

$\mathrm{VR}=0.0$

DO $\mathrm{G} \$ I \$=1, \mathrm{G} \$ \mathrm{P} \$$

$\operatorname{GWVR}(G \$ I \$)=0.0 \mathrm{dO}$

END DO

$\mathrm{VU}=0.0$

$\mathrm{DO} \mathrm{G} \$ \mathrm{I} \$=1, \mathrm{G} \$ \mathrm{P} \$$

$G \$ V U(G \$ I \$)=0.0 \mathrm{~d} 0$

END DO

IF (I.GE. 1 .AND. J.GE. 1) THEN

C

$v=x(k)$

DO $G \$ I \$=1, G \$ P \$$

$G \$ V(G \$ I \$)=G \$ X(G \$ I, K)$

END DO

$V=X(K)$

END IF

IF (I.LT.NX .AND. J.GT.0) THEH

C

$v r=x(k+1)$

DO $G \$ I \$=1, G \$ P \$$

$G \$ V R(G \$ I \$)=G \$ X(G \$ I \$, K+1)$

END DO

$V R=X(K+1)$

END IF

IF (I.GT.O .AND. J.LT.NY) THEN

C

$v u=x(k+n x)$

DO $G \$ I \$=1, G \$ P \$$

$\mathrm{G} \$ V U(G \$ I \$)=G \$ X(G \$ I \$, K+N X)$

END DO

$V U=X(X+N X)$

END IF

C

fquad $=$ fquad + hyx $*(v r-v) * 2+h x y *(v u-v) * * 2$

$D \$ O=V R-V$

$D \$ 4=V U-V$

$D O G \$ I \$=1, G \$ P \$$

$G \$ F Q U A D(G \$ I \$)=G \$ F Q U A D(G \$ I \$)+H Y X * 2 * D \$ O * G \$ V R(G \$ I \$)+$

$+$

$+$

END DO $(-(H X Y * 2 * D \$ 4)-(H Y X * 2 * D \$ 0)) *$

FQUAD $=$ FQUAD + HYX*D\$O**2+HXY*D\$4*2

10 CONTINUE

99999 CONTINUE

20 CONTINUE

99998 CONTINUE

DO $99996 \mathrm{~J}=1, \mathrm{NY}+1$

DO $99997 I=1, N X+1$

$K=N X *(J-1)+I$

$V=0.0$

$D O G \$ I \$=1, G \$ P \$$

$G \$ V(G \$ I \$)=0.0 \mathrm{~d} 0$

END DO

$\mathrm{VL}=0.0$

DO G\$I\$ = 1, G\$P\$

$G \$ V L(G \$ I \$)=0.0 \mathrm{~d} 0$

END DO

$V D=0.0$ 


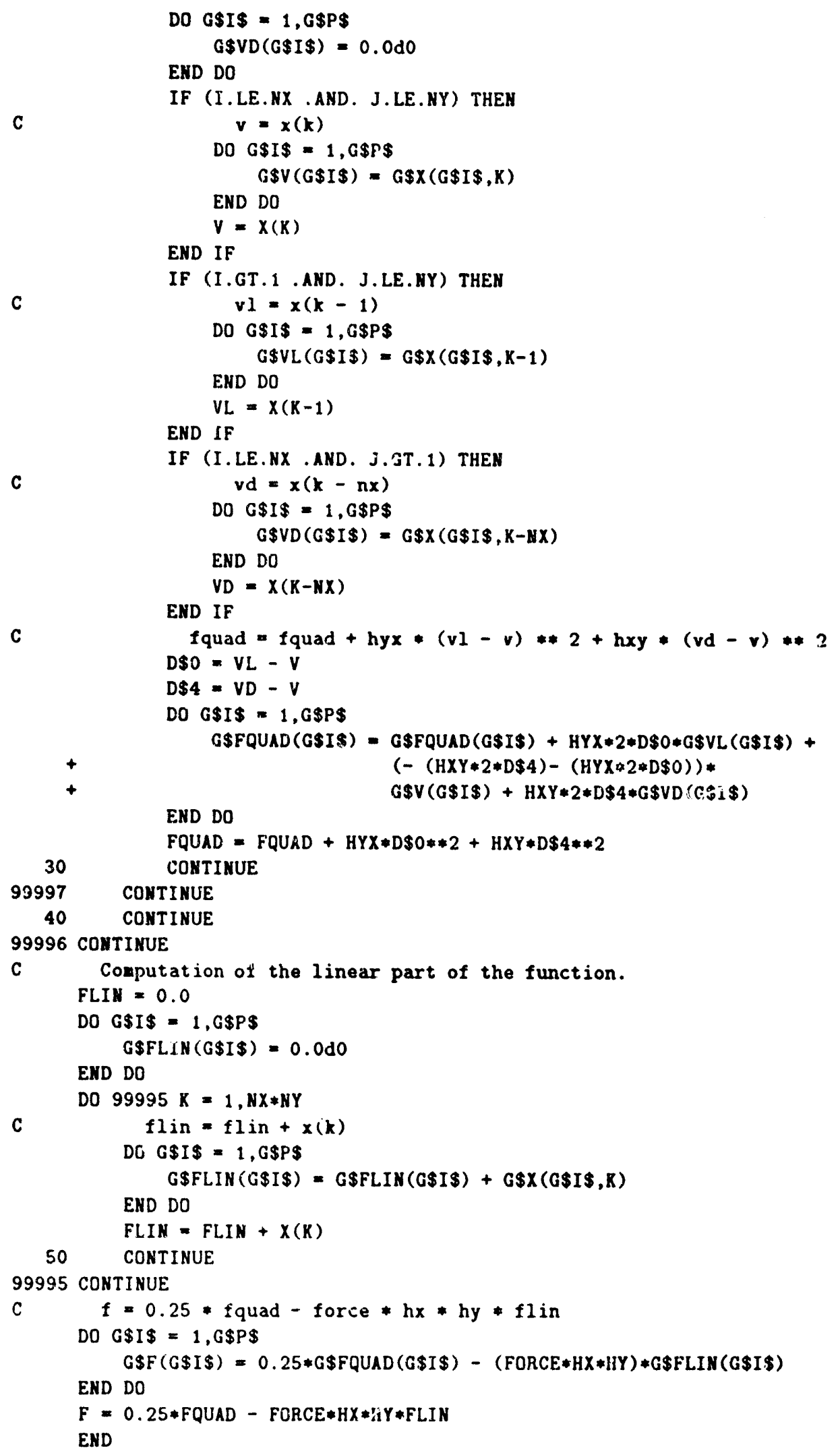


C Reverse Mode for Basic Blocks

SUBROUTINE REV 1 (G\$P\$,N, X, G\$X,LDG $\$ X, F, G \$ F, L D G \$ F, M X, M Y, H X, H Y, F O R C E)$

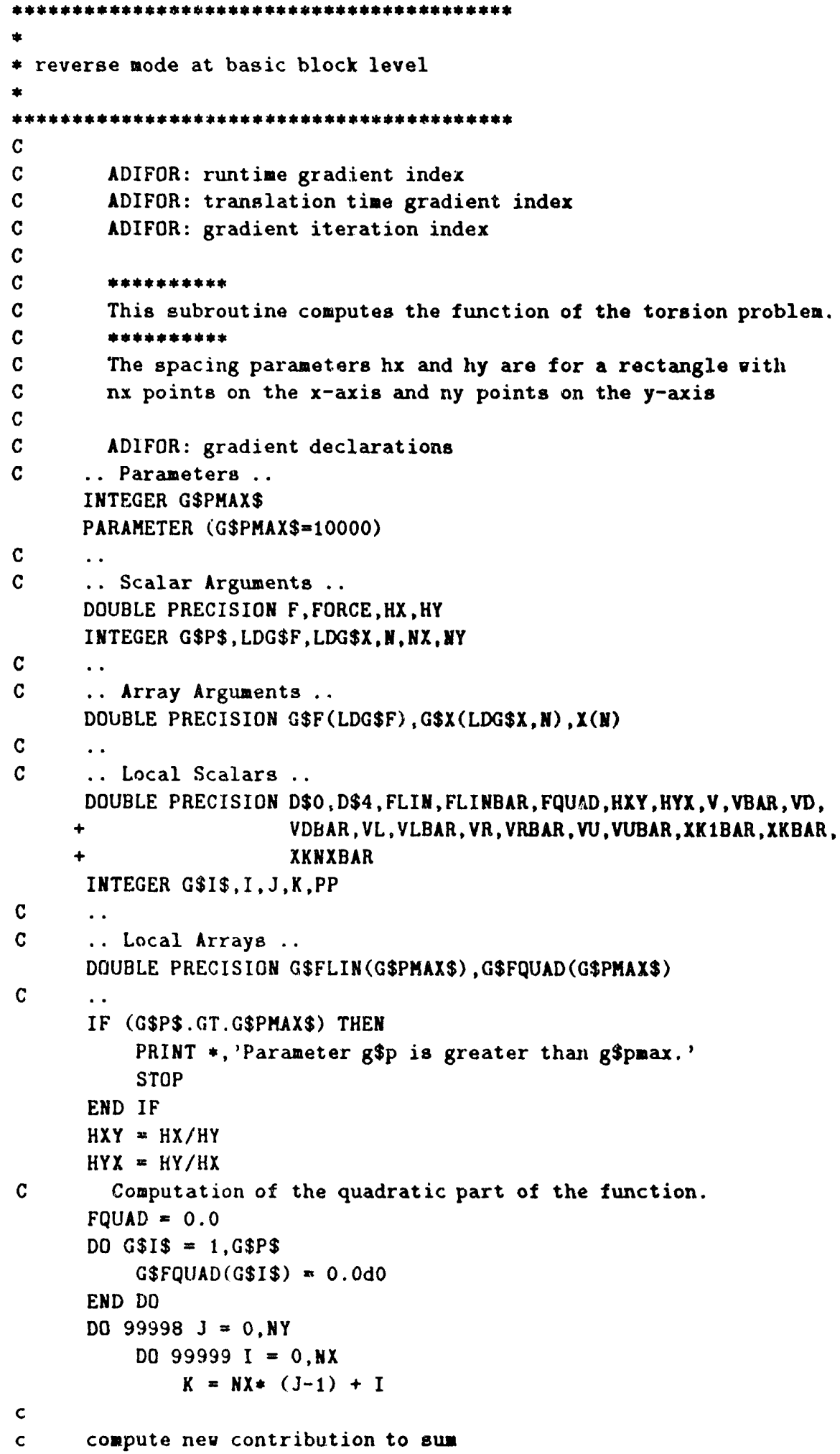




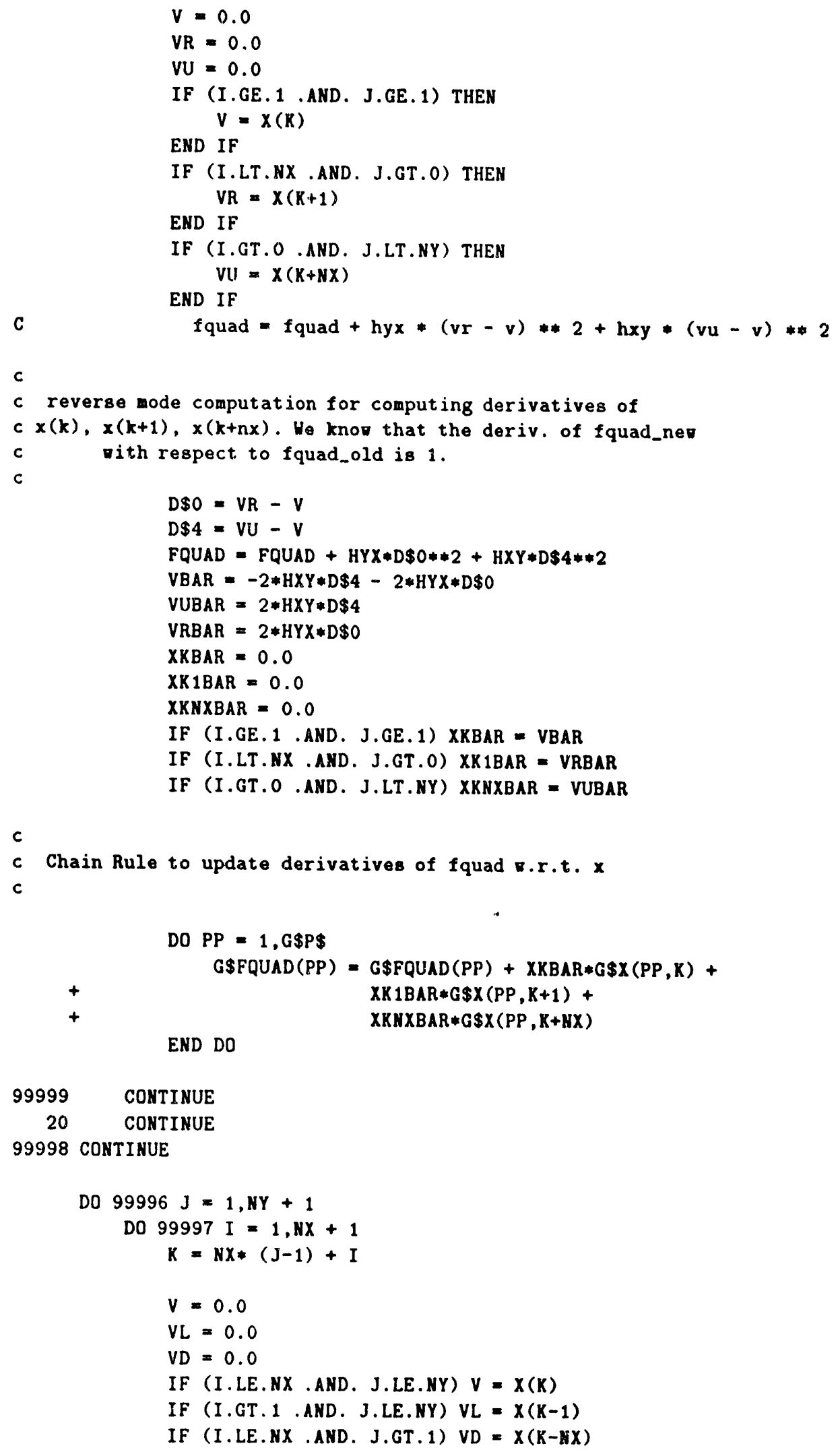


$D \$ O=V L-V$

$D \$ 4=V D-V$

FQUAD $=$ FQUAD + HYX*D\$O**2+HXY*D\$4**2

VBAR $=-2 * H X Y * D \$ 4-2 * H Y X * D \$ 0$

VDBAR $=2 * H X Y * D \$ 4$

VLBAR $=2 * H Y X * D \$ 0$

$X K B A R=0.0$

$X K 1 B A R=0.0$

XKNXBAR $=0.0$

IF (I.LE.NX .AND. J.LE.NY) XKBAR = VBAR

IF (I.GT.1 .AND. J.LE.NY) XR1BAR = VLBAR

IF (I.LE.NX .AND. J.GT.1) XKNXBAR = VDBAR

DO $P P=1, G \$ P \$$

G\$FQUAD (PP) $=$ G\$FQUAD $(P P)+X K B A R * G \$ X(P P, K)+$

$+$ $X K 1 B A R * G \$ X(P P, K-1)+$

$+$

END DO

$X K N X B A R * G \$ X(P P, K-N X)$

99997 CONTINUE

99996 CONTINUE

C

Computation of the linear part of the function.

FLIN $=0.0$

$D D G \$ I \$=1, G \$ P \$$

G\$FLIN (G\$I\$) $=0.0 \mathrm{~d} 0$

END DO

DO $99995 K=1, N X * N Y$

Cm

$g \$ f l i n(k)=g \$ f l i n(k)+1$

FLINBAR $=1.0$

DD $I=1, G \$ P \$$

$\operatorname{G\$ FLIN}(I)=\operatorname{GSFLIN}(I)+\operatorname{FLINBAR} * G \$ X(I, K)$

END DO

FLIN $=$ FLIN $+\chi(K)$

99995 CONTINUE

C

$f=0.25 *$ fquad - force * hx *hy * flin

$D O G \$ I \$=1, G \$ P \$$

$G \$ F(G \$ I \$)=0.25 * G \$ F Q U A D(G \$ I \$)-(F O R C E * H X * H Y) * G \$ F L I N(G \$ I \$)$

END DO

$F=0.25 *$ FQUAD - FORCE*HX*HY*FLIN

END 
D Reverse Mode for Loop Bodies

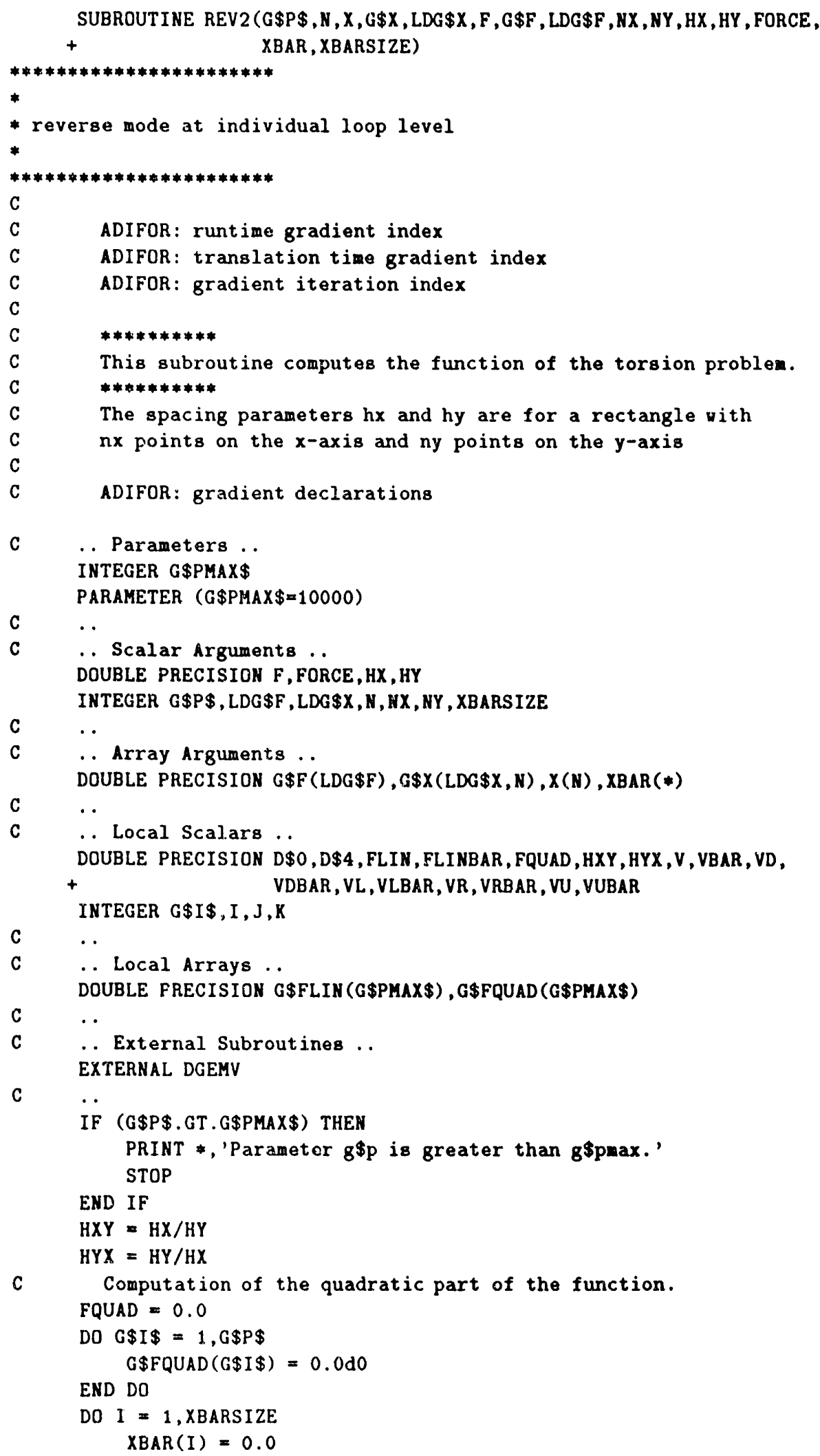




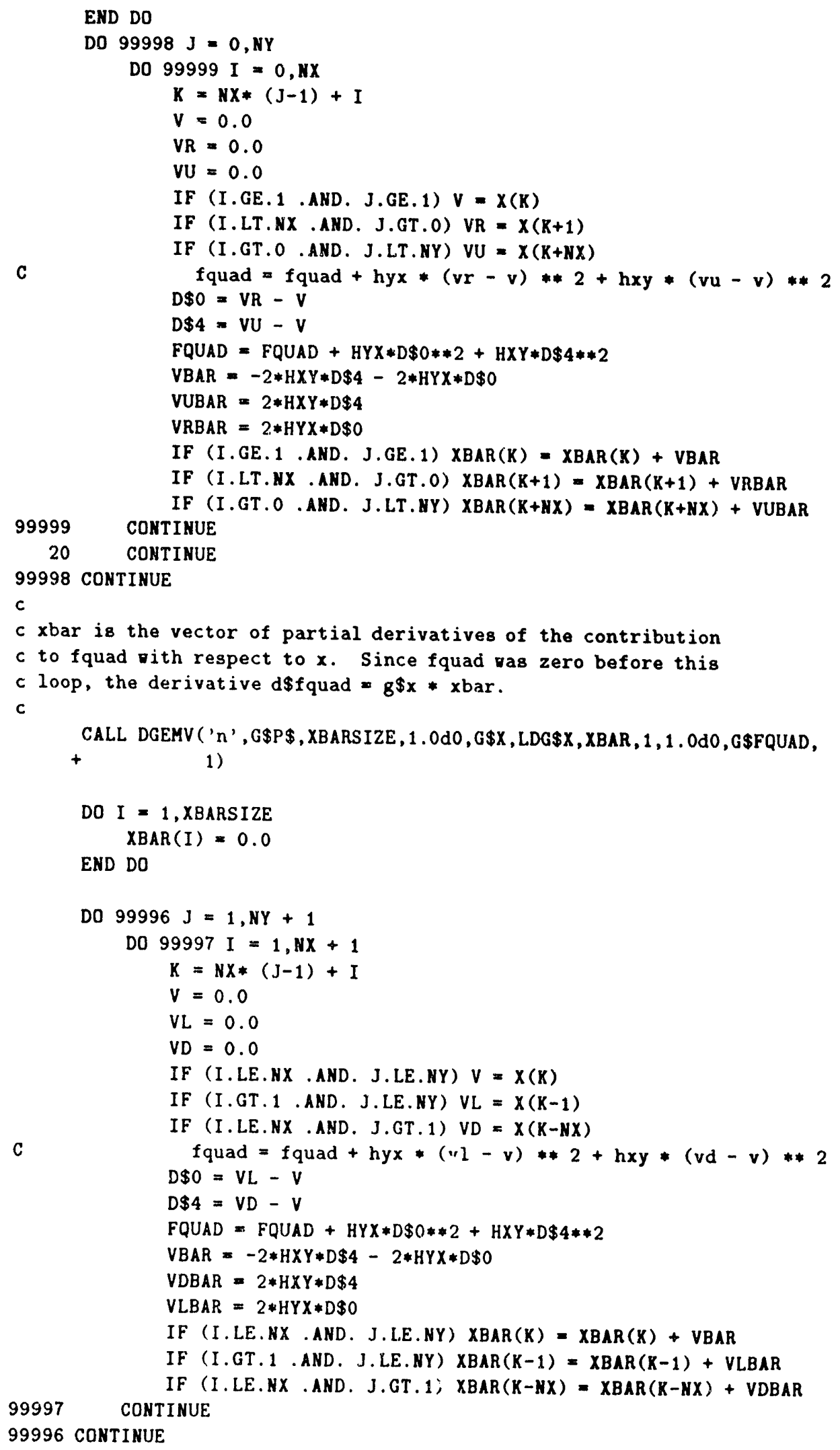


c xbar is the vector of partial derivatives of the contribution

$c$ to fquad with respect to $x$. Since fquad vas already initialized before this loop, the derivative $d \$ f q u a d=d \$ f q u a d+g \$ x * x b a r$.

CALL DGEMV ('n', G\$P\$, XBARSIZE , 1.0d0, G\$X,LDG $\$ X, X B A R, 1,1.0 \mathrm{~d} 0$, G $\$ F Q U A D$, +1 )

C Computation of the linear part of the function.

FLIN $=0.0$

DO G\$I\$ = $1, G \$ P \$$

$G \$ F L I N(G \$ I \$)=0.0 \mathrm{~d} 0$

END DO

DO $I=1, X B A R S I Z E$

$\operatorname{XBAR}(I)=0.0$

END DO

DO $99995 \mathrm{~K}=1, \mathrm{NX} * \mathrm{NY}$

c

$g \$ f l i n(k)=g \$ f \ln (k)+1$

FLINBAR $=1.0$

XBAR $(K)=\operatorname{XBAR}(K)+$ FLINBAR

FLIN $=$ FLIN $+X(K)$

99995 CONTINUE

c

again, $d \$ f q u a d=d \$ f q u a d+g \$ x * x b a r$.

CALL DGEMV ('n', G\$P\$, XBARSIZE, 1.0d0, G\$X, LDG $\$ X, X B A R, 1,1.0 \mathrm{dO}$, G $\$ F L I N$, +1 )

$C \quad f=0.25 *$ fquad - force *hx*hy*flin

$D O G \$ I \$=1, G \$ P \$$

$G \$ F(G \$ I \$)=0.25 * G \$ F Q U A D(G \$ I \$)-(F O R C E * H X * H Y) * G \$ F L I N(G \$ I \$)$

END DO

$F=0.25 * F Q U A D-F O R C E * H X * H Y * F L I N$

END 
E Reverse Mode for the Whole Program

SUBROUTINE REV 3 (N , X , F , NX , NY , HX , HY , FORCE, XBAR, XBAFSIZE)

c

C ADIFOR: runtime gradient index

C ADIFOR: translation time gradient index

C

C

C

C

C

$\mathrm{C}$

$\mathrm{C}$

C

C

************1\%

This subroutine computes the function of the torsion problem.

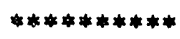

The spacing parameters $h x$ and hy are for a rectangle with

$n x$ points on the $x$-axis and ny points on the y-axis

ADIFOR: gradient declarations

.. Scalar Arguments ..

DOUBLE PRECISION F, FORCE, HX, HY

INTEGER N, NX, NY , XBARSIZE

C

.

.. Array Arguments .

DOUBLE PRECISION $X(N), X B A R(*)$

C .

C .. Local Scalars .. DOURLE PRECISION

$+$

INTEGER I, J,K

VDBAR, VL, VLBAK, VR, VRBAR, VU, VUBAR

C

$H X Y=H X / H Y$

$\mathrm{HYX}=\mathrm{HY} / \mathrm{HX}$

C

Computation of the quadratic part of the function.

FQUAD $=0.0$

DO $I=1, X B A R S I Z E$

$\operatorname{XBAR}(I)=0.0$

END DO

DO $99998 \mathrm{~J}=0, \mathrm{NY}$

DO $99999 I=0, N X$

$K=N X *(J-1)+I$

$V=0.0$

$\mathrm{VR}=0.0$

$\mathrm{VU}=0.0$

IF (I.GE. 1 .AND. J.GE.1) $V=X(K)$

IF (I.LT.NX .AND. J.GT.0) VR $=X(K+1)$

IF (I.GT.0 .AND. J.LT.NY) VU $=X(K+N X)$

C

fquad $=$ fquad + hyx $*(v r-v) * * 2+h x y *(v u-v) * * 2$

$D \$ O=V R-V$

$D \$ 4=V U-V$

FQUAD $=$ FQUAD + HYX*D\$O**2+HXY*D\$4**2

VBAR $=-2 * H X Y * D \$ 4-2 * H Y X * D \$ 0$

VUBAR $=2 * H X Y * D \$ 4$

VRBAR $=2 * H Y X * D \$ 0$

IF (I.GE. 1 .AND. J.GE.1) $\mathrm{XBAR}(\mathrm{K})=\mathrm{XBAR}(\mathrm{K})+\mathrm{VBAR}$

IF (I.LT.NX .AND. J.GT.0) XBAR $(K+1)=X B A R(K+1)+$ VRBAR

99999

IF (I.GT.O .AND. J.LT.NY) XBAR $(K+N X)=X B A R(K+N X)+$ VUBAR

99998 CONTINUE

DO $99996 \mathrm{~J}=1, \mathrm{NY}+1$ 


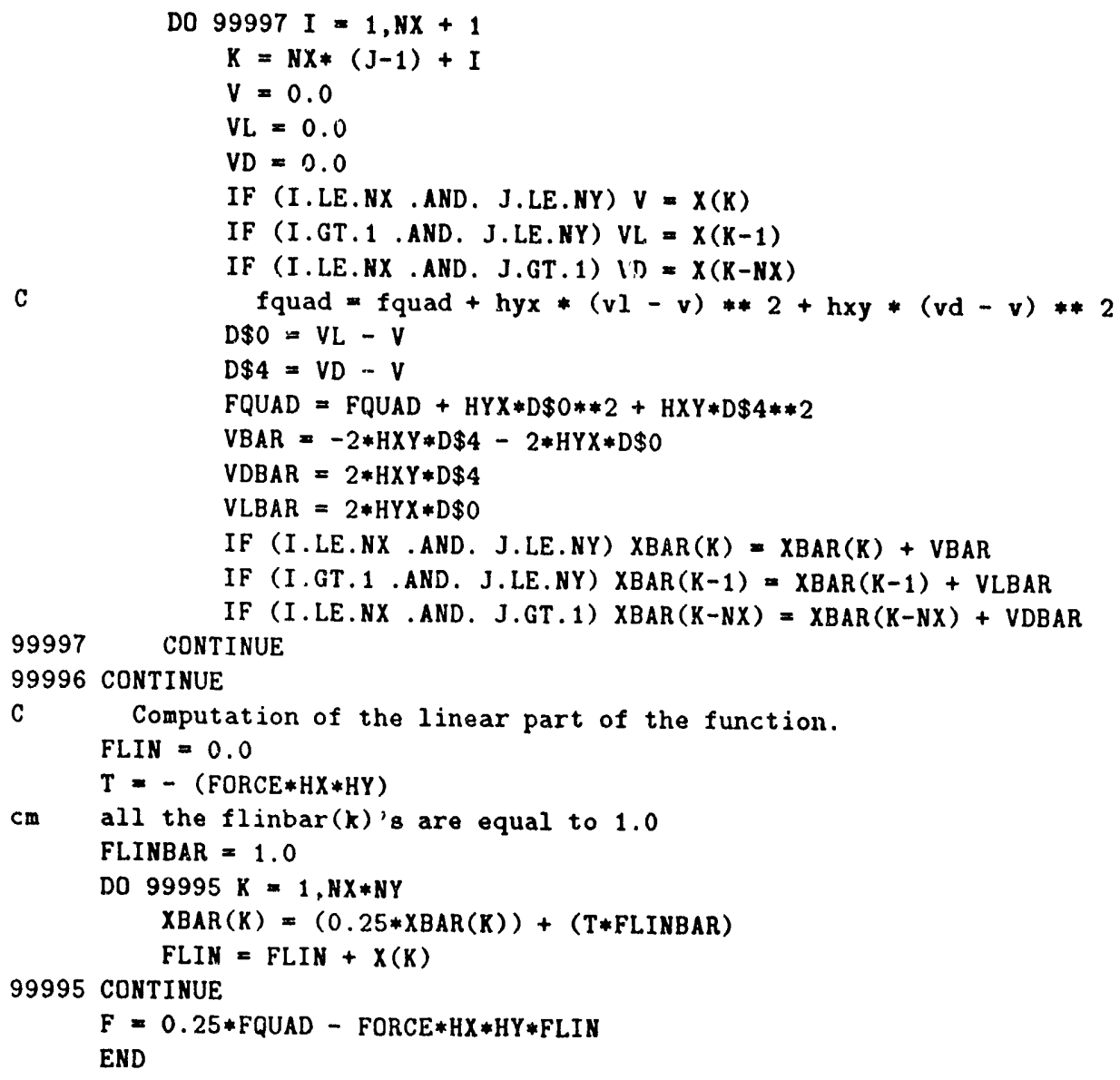


F Derivative Code for Component Functions

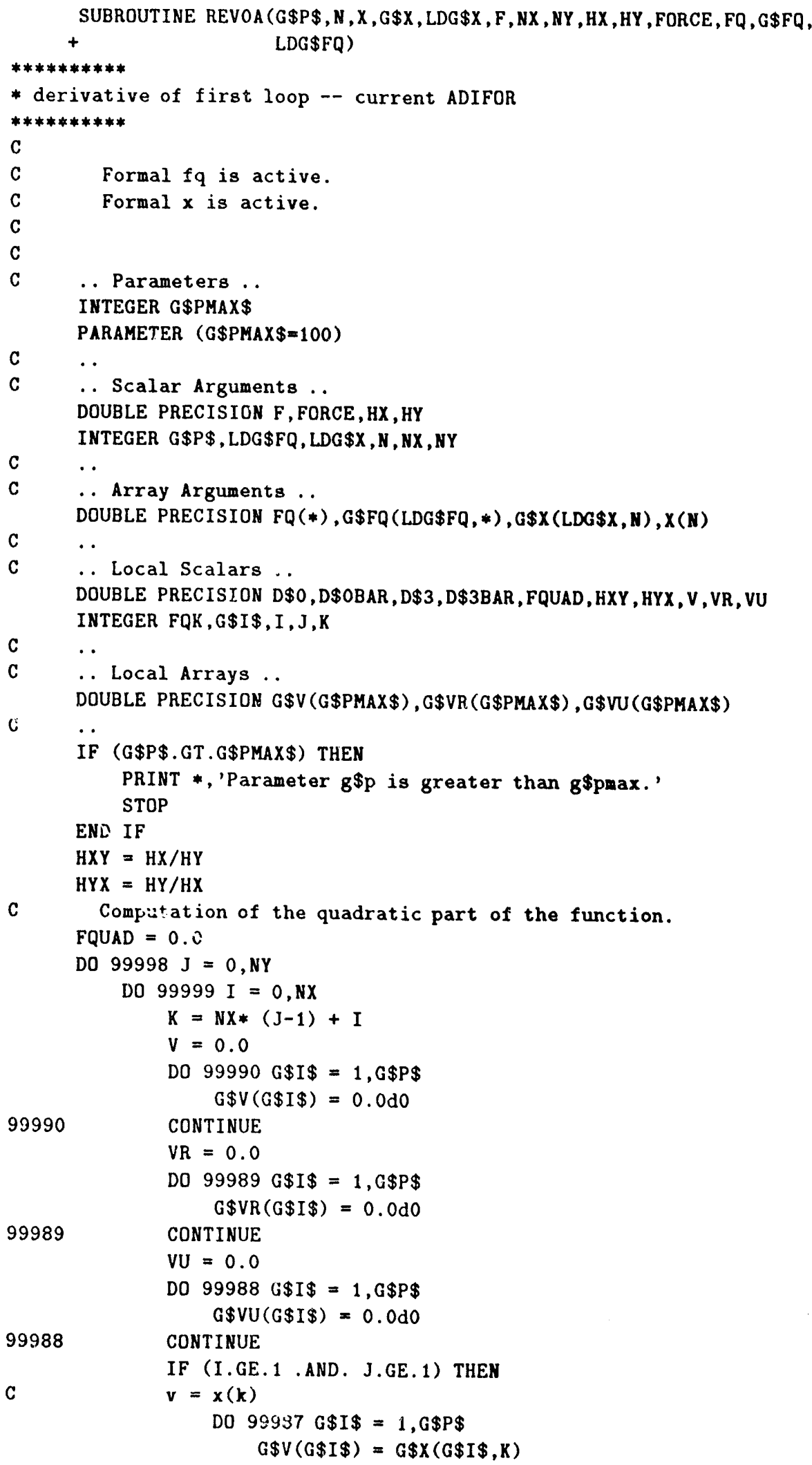




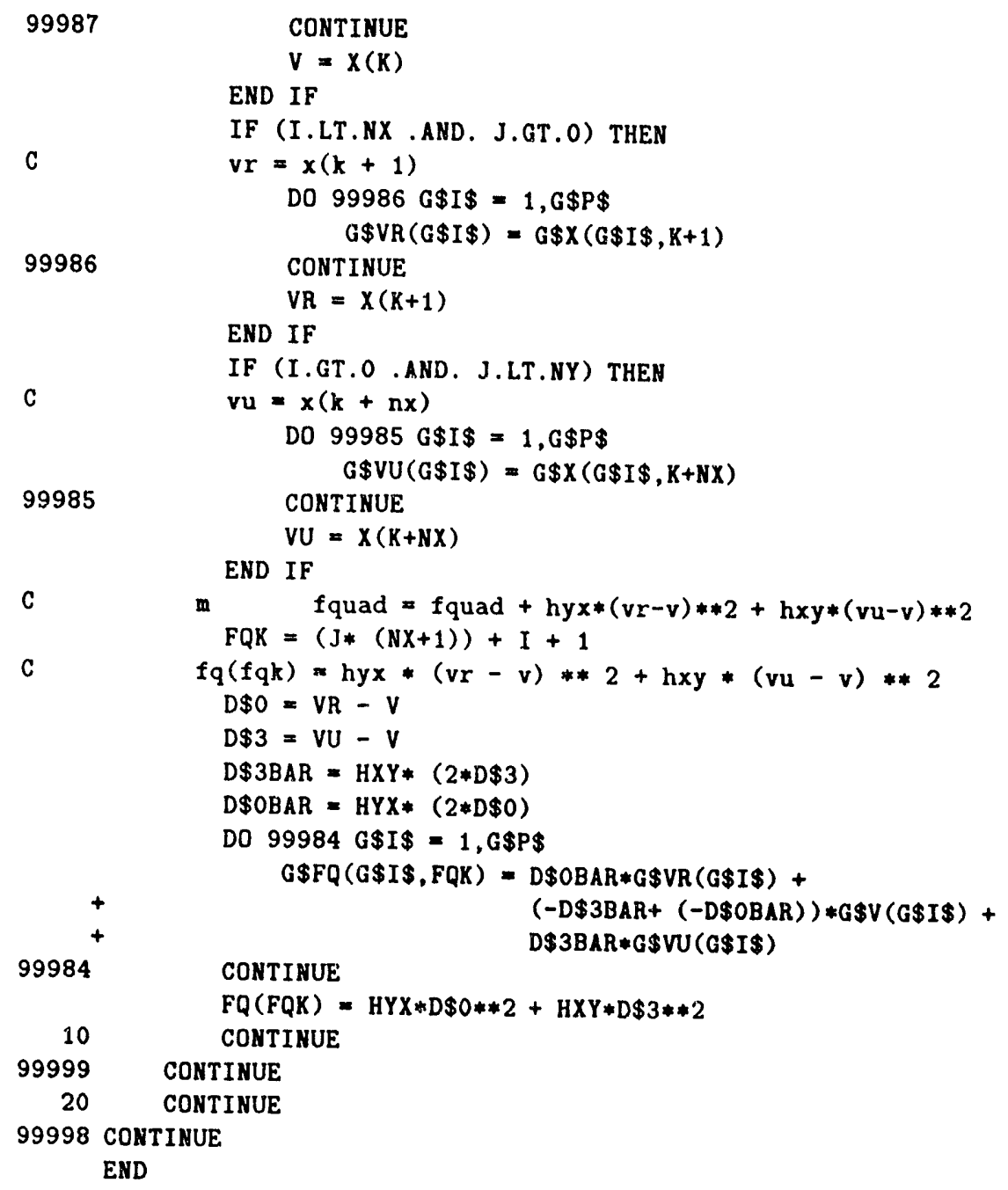


SUBROUTINE REVOB(G\$P\$, $N, X, G \$ X, L D G \$ X, F, N X, N Y, H X, H Y, F O R C E, F Q Q, G \$ F Q Q$,

\section{$+$ LDG $\$ F Q Q)$}

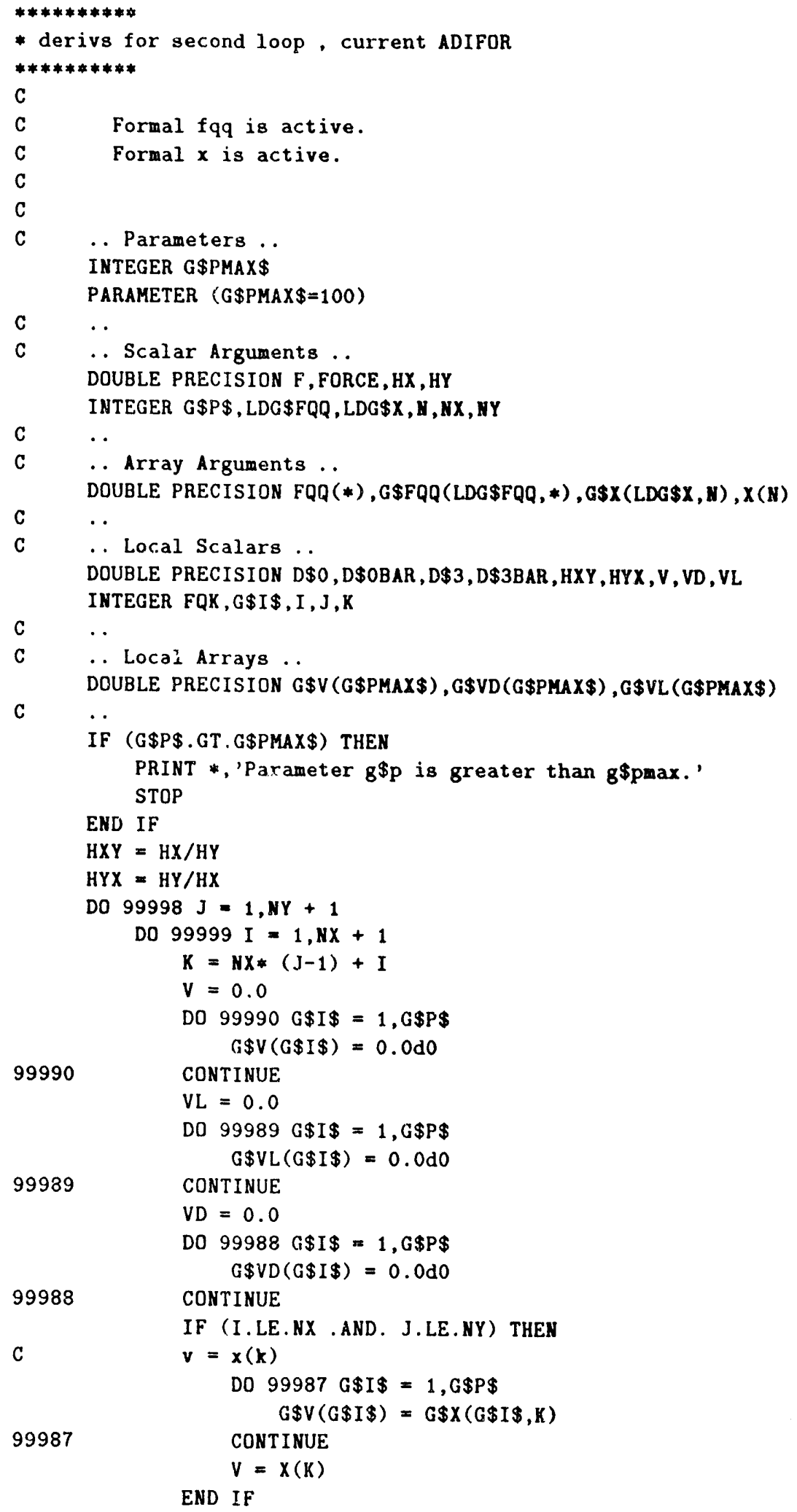


C

99986

C

99985

C

C END IF

IF (I.LE.NX .AND. J.GT.1) THEN

$\mathrm{vd}=\mathrm{x}(\mathrm{k}-\mathrm{nx})$

DO 99985 G $\$ I \$=1, G \$ P \$$

$\mathrm{G} \$ V D(G \$ I \$)=G \$ X(G \$ I \$, K-N X)$

CONTINUE

$V D=X(K-N X)$

END IF

fquad $=$ fquad + hyx $*(v l-v) * * 2+h x y *(v d-v) * * 2$

$F Q K=((J-1) *(N X+1))+I$

$f q q(f q k)=$ hyx $*(v l-v) * * 2+h x y *(v d-v) * * 2$

$D \$ 0=V L-V$

$D \$ 3=V D-V$

$D \$ 3 B A R=H X Y *(2 * D \$ 3)$

$D \$ O B A R=H Y X *(2 * D \$ 0)$

DO 99984 G\$I\$ = $1, G \$ P \$$

$G \$ F Q Q(G \$ I \$, F Q K)=D \$ O B A R * G \$ V L(G \$ I \$)+$

$(-D \$ 3 B A R+(-D \$ O B A R)) * G \$ V(G \$ I \$)+$

CONTINUE

$D \$ 3 B A R * G \$ V D(G \$ I)$

99984

30

99999

40

99998

END

$F Q Q(F Q K)=H Y X * D \$ 0 * * 2+H X Y * D \$ 3 * * 2$

CONTINUE

CONT INUE

CONTINUE

CONTINUE 
SUBROUTINE REV 1C (G\$P\$, N, X,G\$X,LDG $\$ X, F, N X, N Y, H X, H Y, F O R C E, F P, R G F P$,

\section{$+\quad$ LDG\$FP)}

\section{$* * * * * * * n * * * * * * *$}

* third loop, basic block reverse mode

$* * * * * * * * * * * * * * *$

C .. Scalar Arguments ..

DOUBLE PRECISION F, FORCE, $\mathrm{HX}, \mathrm{HY}$

INTEGER G\$P\$, LDG\$FP, LDG\$X,N,NX,NY

C $\quad$.

C .. Array Arguments ..

DOUBLE PRECISION FP $(*), G \$ X(\operatorname{LGG} \$ X, *), \operatorname{RGFP}(\operatorname{LDG} \$ F P, *), X(N)$

C . .

C .. Local Scalars.. DOUBLE PRECISION FPBAR, HXY, HYX

INTEGER I, K

C

$\mathrm{HXY}=\mathrm{HX} / \mathrm{HY}$

$\mathrm{HYX}=\mathrm{HY} / \mathrm{HX}$

c Computation of the linear part of the function.

DO $50 K=1, N X * N Y$

FPBAR $=1.0$

$\operatorname{RGFP}(1, K)=0.0$

DO $I=1, G \$ P \$$

END DO

$\operatorname{RGFP}(I, K)=\operatorname{RGFP}(I, K)+\operatorname{FPBAR} * G \$ X(I, K)$

$F P(K)=X(K)$

50 CONTINUE

END 
G Enhanced Derivative Code for Component Functions

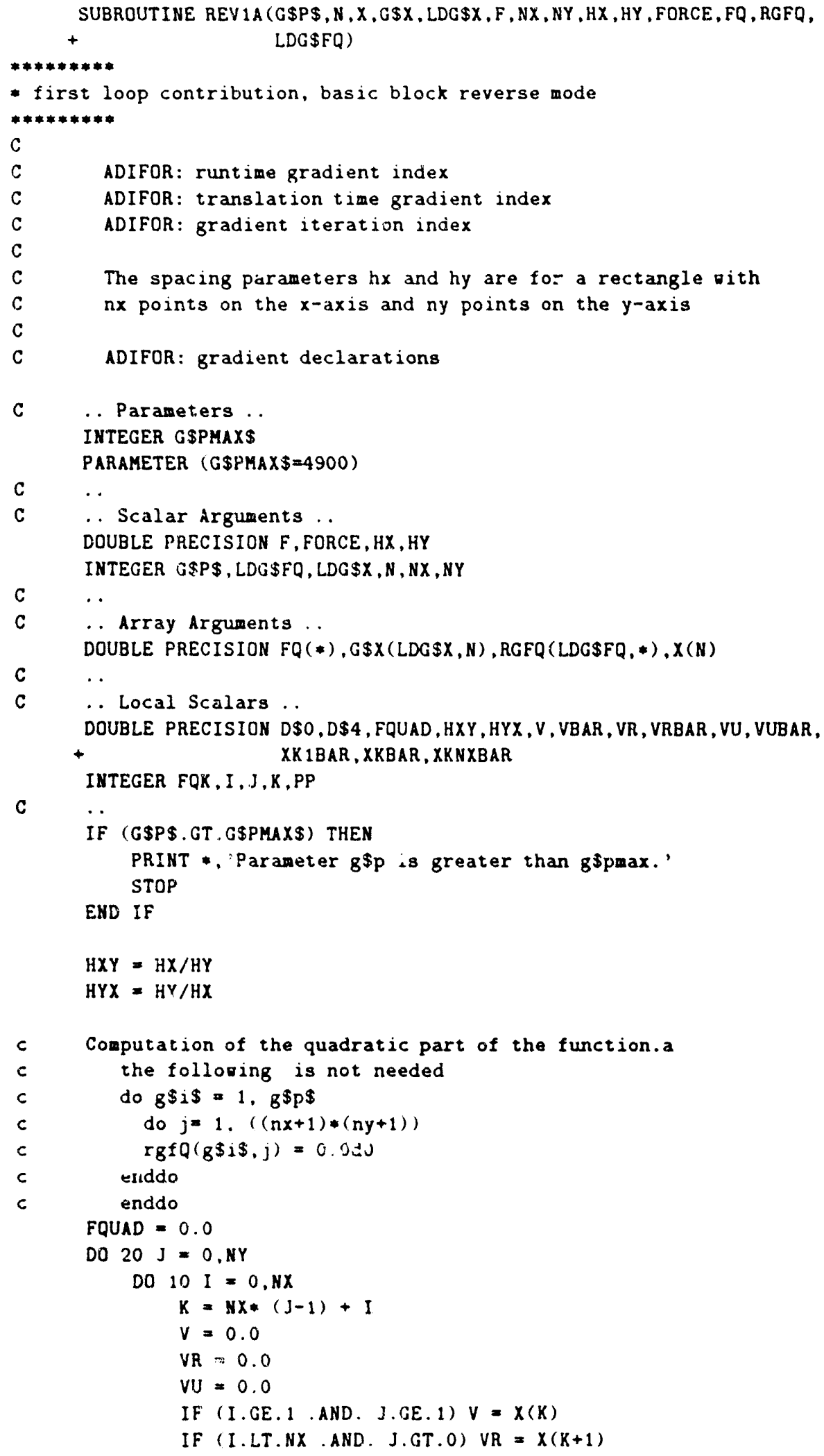




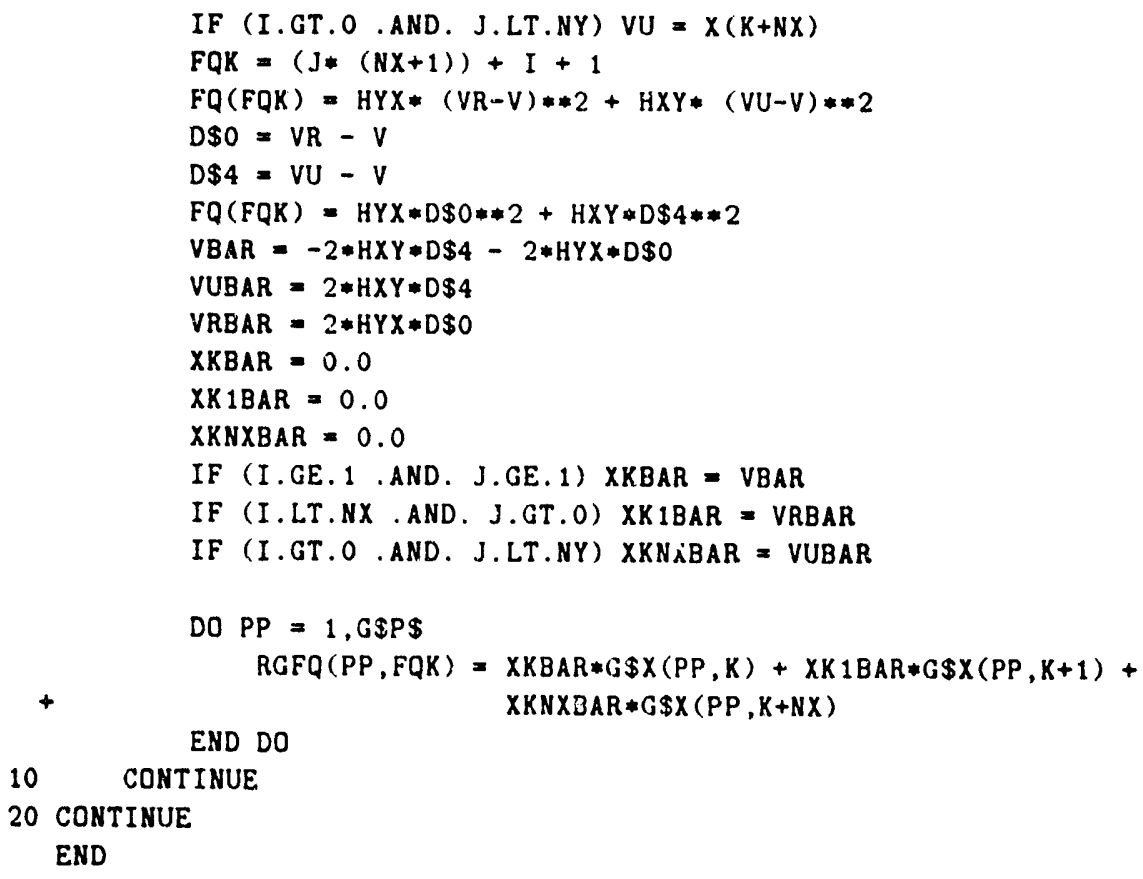


SUBROUTINE REV1B (G\$P\$, $N, X, G \$ X, L D G \$ X, F, N X, N Y, H X, H Y$, FORCE, FQQ , RGFQQ,

\section{$+\quad$ LDG\$FQQ}

* second loop, basic block reverse mode

***********4)

C

C ADIFOR: runtime gradient index

ADIFOR: translation time gradient index

ADIFOR: gradient iteration index

The spacing parameters $h x$ and hy are for a rectangle with

$n x$ points on the $x$-axis and ny points on the $y$-axis

ADIFOR: gradient declarations

C ..Parameters.

INTEGER G\$PMAX\$

PARAMETER (G\$PMAX $\$=4900)$

C $\quad$.

C .. Scalar Arguments ..

DOUBLE PRECISION F, FORCE, HX, HY

INTEGER G\$P\$, LDG\$FQQ, LDG\$X, N, NY, NY

C $\quad$.

C .. Array Arguments ..

DOUBLE PRECISION FQQ (*), G\$X (LDG $\$ X, N), \operatorname{RGFQQ(LDG\$ FQQ,*),X(N)}$

C $\cdots$

C .. Local Scalars..

DOUBLE PRECISION D\$O,D\$4, FQUAD, HXY, HYX, V , VBAR, VD, VDBAR, VL, VLBAR,

$+$ XK 1BAR, XKBAR, XKNXBAR

INTEGER FQK $, I, J, K, P P$

C

IF (G\$P\$.GT.G\$PMAX\$) THEN

PRINT *,'Parameter $8 \$ p$ is greater than $g \$ p$ max.' STOP

END IF

$H X Y=H X / H Y$

$\mathrm{HYX}=\mathrm{HY} / \mathrm{HX}$

Computation of the quadratic part of the function. the folloring is NOT needed

do $g \$ i \$=1, g \$ p \$$

do $j=1,((n x+1) *(n y+1))$

$\operatorname{rgfQQ}(g \$ i \$, j)=0.0 \mathrm{dO}$

enddo

enddo

FQUAD $=0.0$

DO $40 \mathrm{~J}=1, N Y+1$

DO $30 I=1, N X+1$

$K=N X *(J-1)+I$

$V=0.0$

$V L=0.0$

$V D=0.0$

IF (I.LE.NX .AND. J.LE.NY) $V=X(K)$

IF (I.GT.1 AND. J.LE.NY) $V L=X(K-1)$

IF (I.LE.NX .AND. J.GT.1) VD $=X(K-N X)$

$\mathrm{cm}$

fquad $=$ fquad $+h y x *(v 1-v) * * 2+h x y *(v d-v) * 2$ 


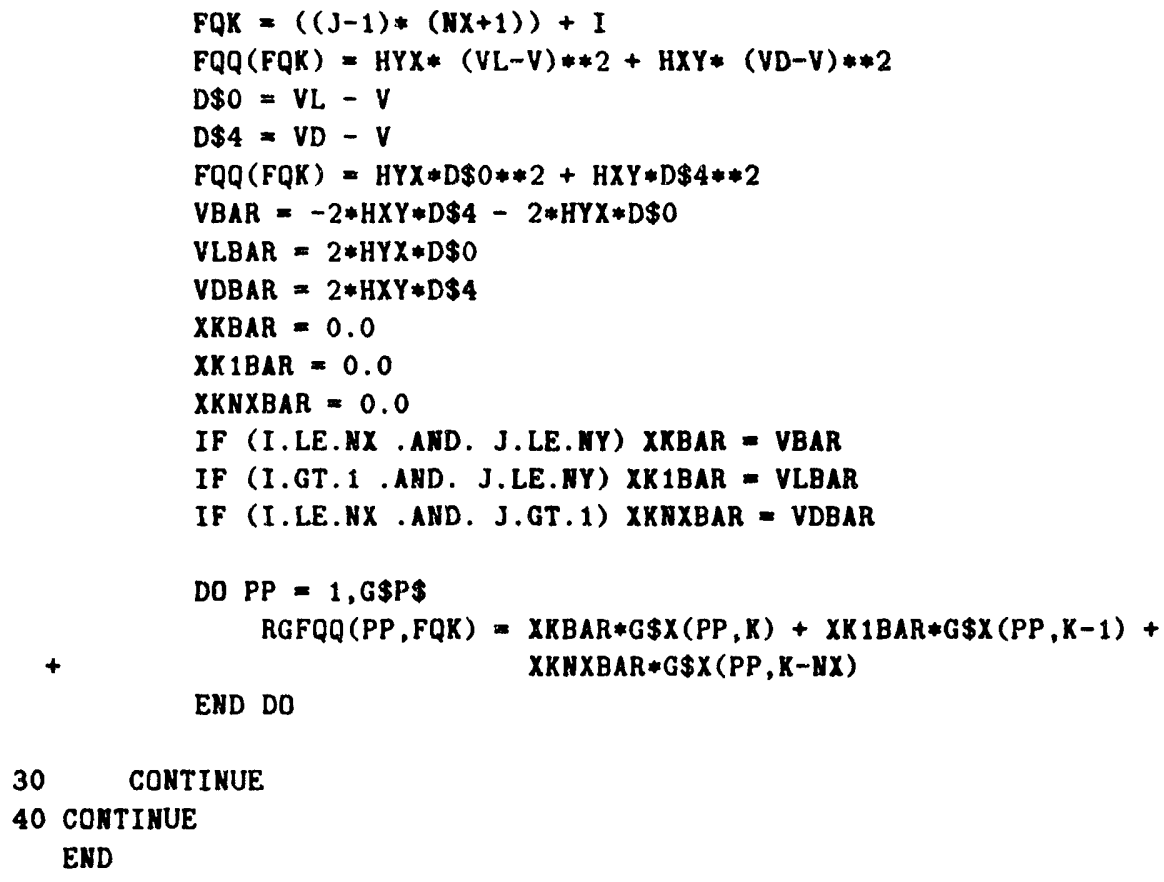


SUBROUTINE REV1C(G\$P\$,N,X,G\$X, LDG $\$ X, F, N X, N Y, H X, H Y, F O R C E, F P, R G F P$,

$+$ LDG\$FP)

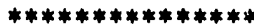

* third loop, basic block reverse mode

$* * * * * * * * * * * * * * *$

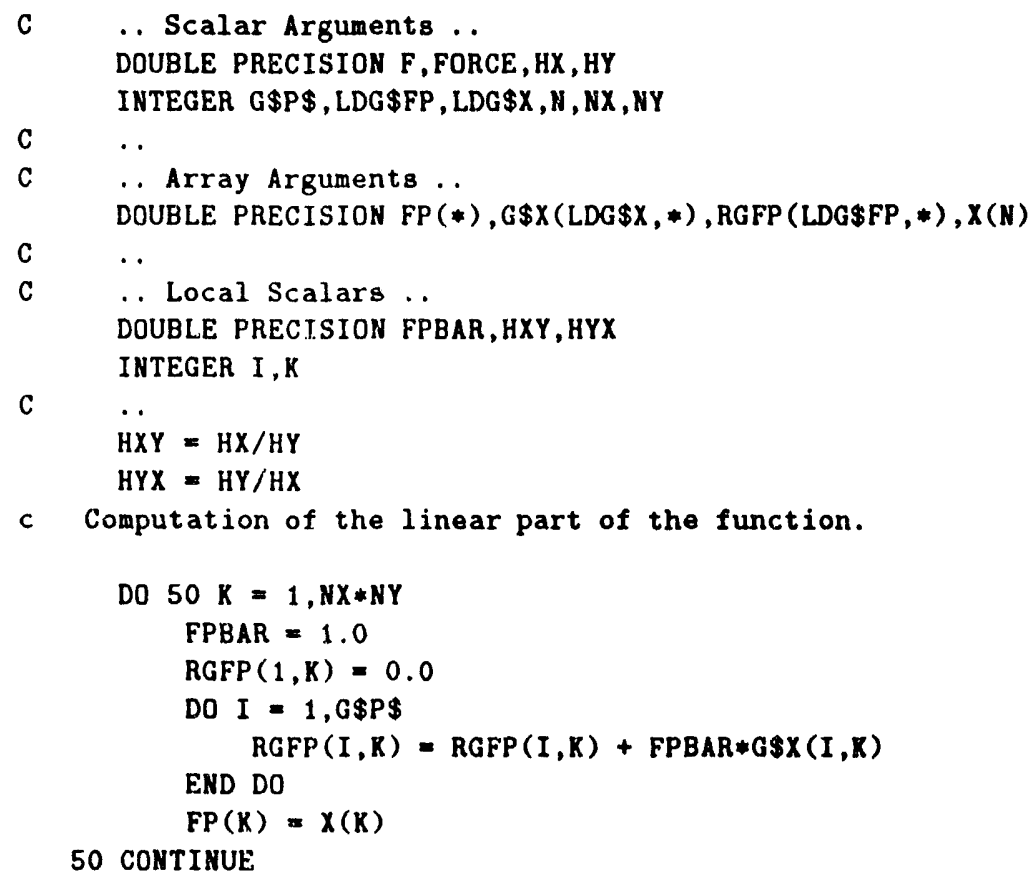

C

$H X Y=H X / H Y$

$H Y X=H Y / H X$

c Computation of the linear part of the function.

DO $50 K=1, N X * N Y$

FPBAR $=1.0$

$\operatorname{RGFP}(1, K)=0.0$

DO $I=1, G \$ P \$$

END DO

END 

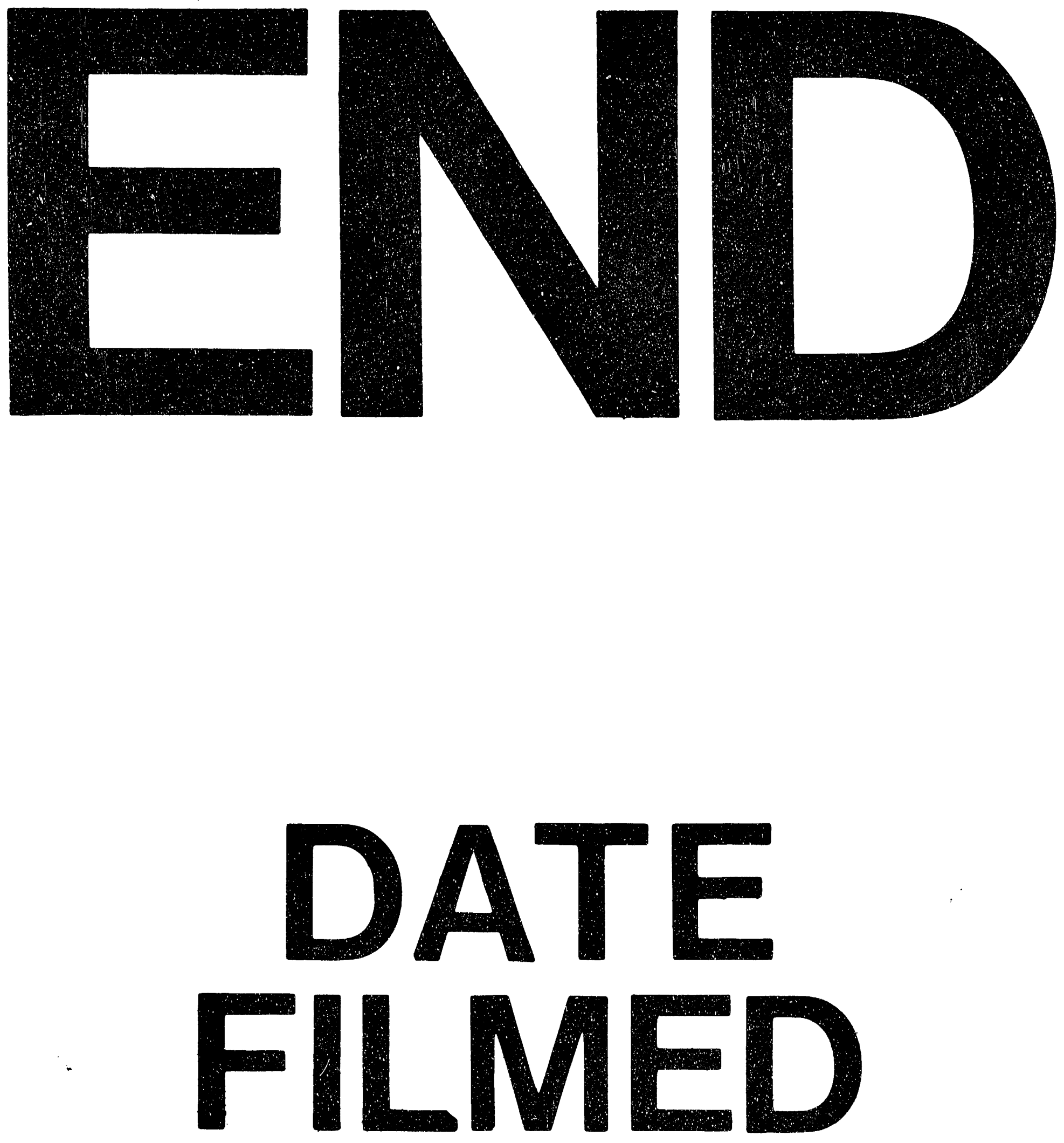

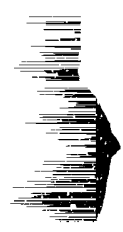

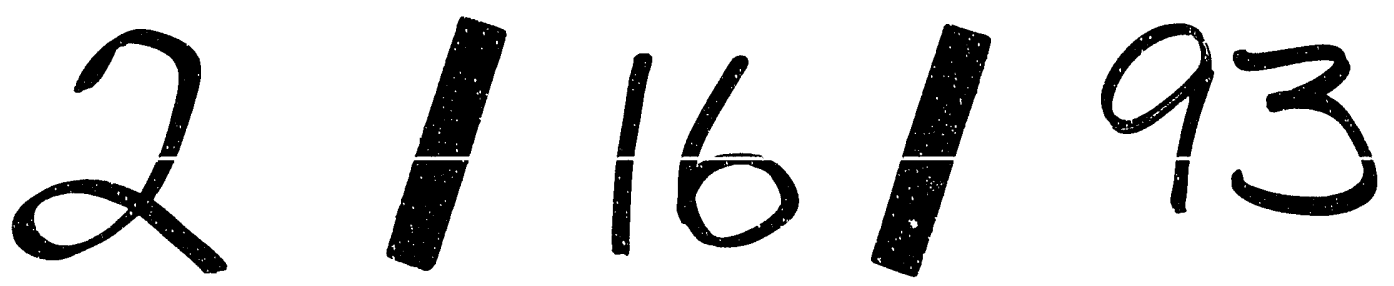


, 